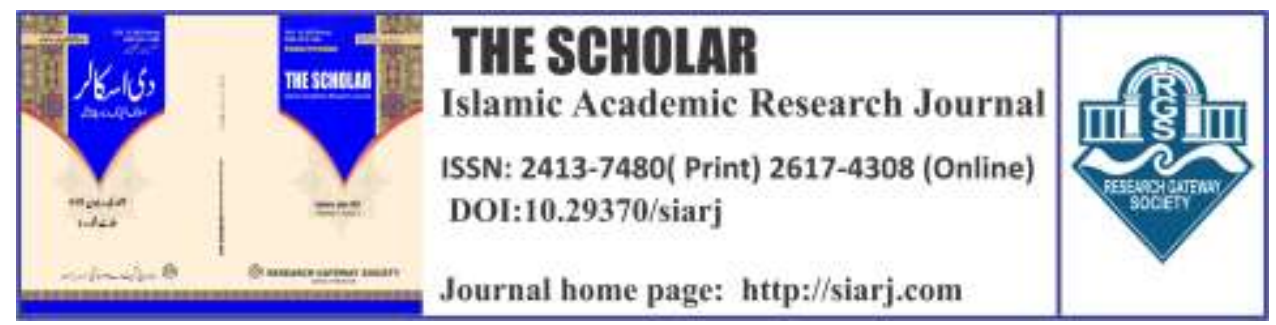

منهجية النقد عند الإمام الدارقطني في كتابه الأفراد والغرائب

$$
\text { (دراسة ونقد) (دمب) }
$$

\title{
THE METHODOLOGY OF CRITICISM ACCORDING TI IMAM AL - DARAQUTNI IN HIS BOOK “AL - AFRAD-WAL - GHRAEB” STUDY AND
}

\section{1.-Umar Hayat}

Ph.D Scholar, Department of Hadith, Fuculty

Usoluddin, International Islamic Universit

Islamabad, Pakistan

Email : umar.hayat3@gmail.com

\section{ORCID ID:}

\section{https://orcid.org/0000-0001-5721-266X}

\section{To cite this article:}

Hayat, Umar, and Abdul Samad Shaikh. "THE METHODOLOGY OF CRITICISM ACCORDING TI

IMAM AL - DARAQUTNI IN HIS BOOK 'AL - AFRAD-WAL - GHRAEB' STUDY AND CRITCISM." The

Scholar-Islamic Academic Research Journal 7, No. 2 (November 20, 2021).

To link to this article: https://doi.org/10.29370/siarj/issue13arabic1

Journal

Publisher

DOI:

URL:

License:

Journal homepage

Published online:

\section{2. - Abdul Samad Shaikh}

Assistant professor, International Islamic

University, Islamabad, Pakistan

Email: abdulsamad.shaikh@iiu.edu.pk

\section{ORCID ID:}

https://orcid.org/0000-0001-5721-266X
The Scholar Islamic Academic Research Journal

Vol. 7, No. 2 || July-December 2020 || P. 1-28

Research Gateway Society

10.29370/siarj/issue13ar1

https://doi.org/10.29370/siarj/ issue13arabic1

Copyright c 2017 NC-SA 4.0

www.siarj.com

2021-11-20
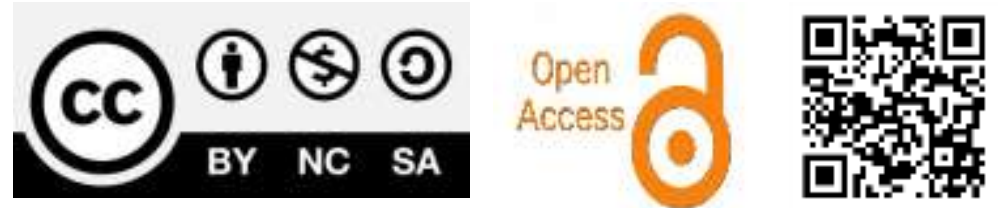


\title{
منهجية النقد عند الإمام الدارقطني في كتابه الأفراد والغرائب \\ (دراسة ونقد (دماسد) \\ THE METHODOLOGY OF CRITICISM ACCORDING TI IMAM AL - DARAQUTNI IN HIS BOOK “AL - AFRAD-WAL - GHRAEB” STUDY \\ AND
}

\author{
U mar H ayat, Abdul Samad Shaikh
}

\begin{abstract}
:
Hadith is the second source of Islamic Law. It occupies central attention generally in religious and particularly inlslamic Studies. It has a number of branches as discipline and the most famous one is the Science of Ilal. It is considered to be the more complicated, therefore; there is a very small number of Hadith Scholars who excel in this branch of Hadith Sciences. Imam Dara Qutni is known for his scholarly contributed work in the Science of Ilal. His book named "Al-Ilal Al-Waridah fi Al-Ahadith AlNabawiyyah" is one of the most studied and discussed book in this regard. Although, Ilal had been discussed before Imam Dara Qutni, but he is one who strived to collect and criticize most of this kind of narrative from Hadith Literature. In this article, the researcher is going to present methodological glimpses from Imam Dara Qutni's less famous book named "Al-Afra'dwa Al-Ghrae'b". This study is important due to unavailability of this book in the Islamic Library and it is indexed in those books which are missing from the traditional literature of Hadith. So this article will help to know the new dimension in the Science of Ilal.
\end{abstract}

KEYWORDS: Ilal, Hadith, Tradition, Science, Islamic, Dara Qutni.

$$
\text { الكلمات المفتاحية: العلل، الحديث، التقليدية، العلم، الإسلام، الدارقطني. }
$$




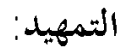

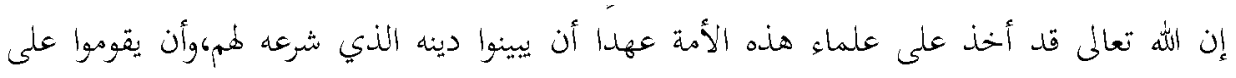

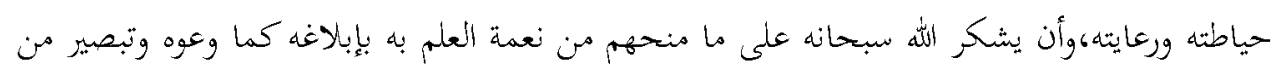

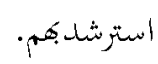
وإن من أجل هذه العلوم علم الحديث النبوي،فهو التالي لعلم القرآن في المنزلة وهو المبين لإجمال القرآن

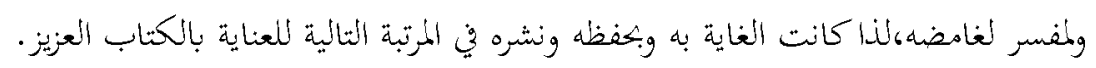

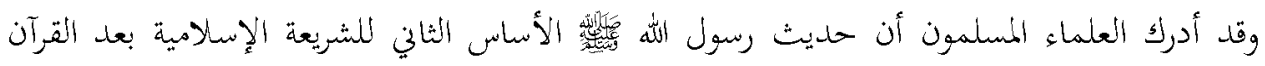
للذلك عكفوا عليه خلدمة لا نظير هلا في الوجود. ومن هئلاء العلماء الإمام الحافظ علي بن عمر أبو الحسن الدارقطني،صاحب النصانيف كثيرة ومفيدة.

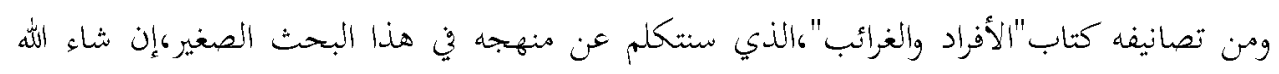
تعالى. أعددت هذا البحث على النقاط التالية:

$$
\text { ثالثان أنعا: التمهيد. }
$$

$$
\text { سابعا: الخاتمة ونتائج البحث. }
$$

\section{ثانيا:تعريف ومعنى المنهج لغة واصطلاحا.}

$$
\text { أذكر أولا تعريف المنهج لغة وهو يدل على الطريق أو أولى السبيل. }
$$

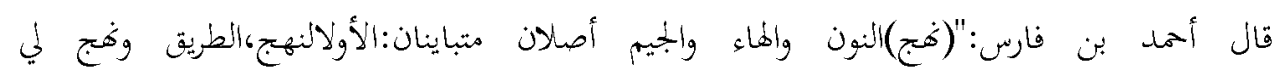

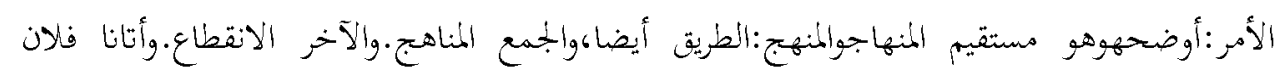




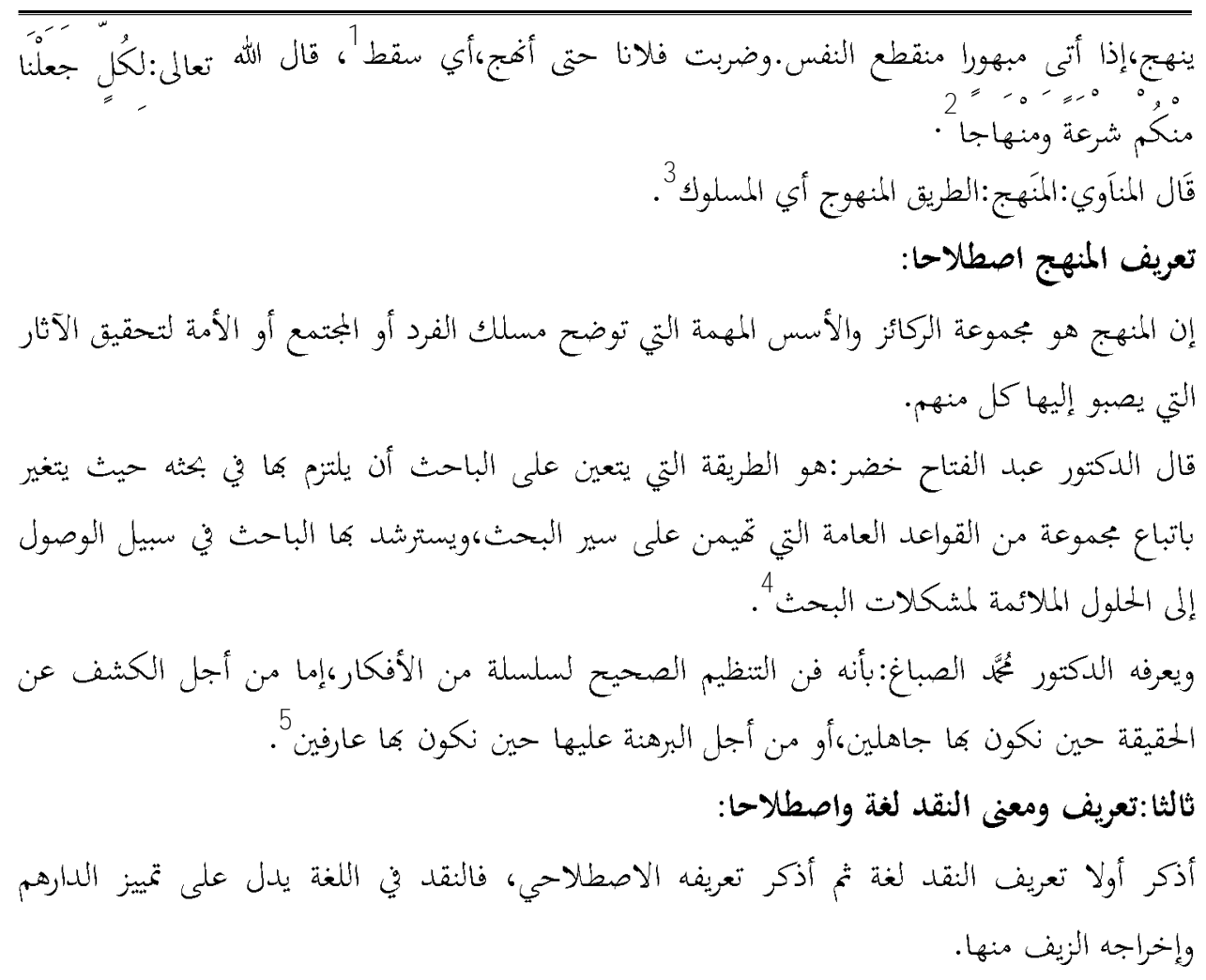

1_ Al- R azi, Ahmad ibn faris, Abu Al- $\mathrm{H}$ asan, M ujam M aqayees Al'lughah, V.4, P.361, D ar A I-Fikar And Al-R agib Al-A sfahani, Al- H usan Ibn M uhammad, A bu Al- Q asim, A/- M ufradat Fee Greeb A /- Q uran, P.825,D ar Al- Q alam, Beirut, 1412 A.H .

2 _Al- Q uran, Surah 5,A yat 48.

3 _ Al- M unavi, M uhammad Ibn Taj Al A rifeen, $A$ /- Tawqeef $A$ la

M uhimmat A/- Ta'areef, V.1, P317, A'alim Al- K utab Abdul K haliq,Sarwat, Al- Q ahirah, 1410 A.H .

4 _ Dr.Abdul Fattah K hazir, Azmina T ul Bahas Al-Elmi, V .2,P.11.

5 _ Dr. M uhammad Al- Sabbagh, $A$ /- $M$ anahij $W$ al A traf $A$ /- Talifiah Fee Turathina, V.1, P.9. 


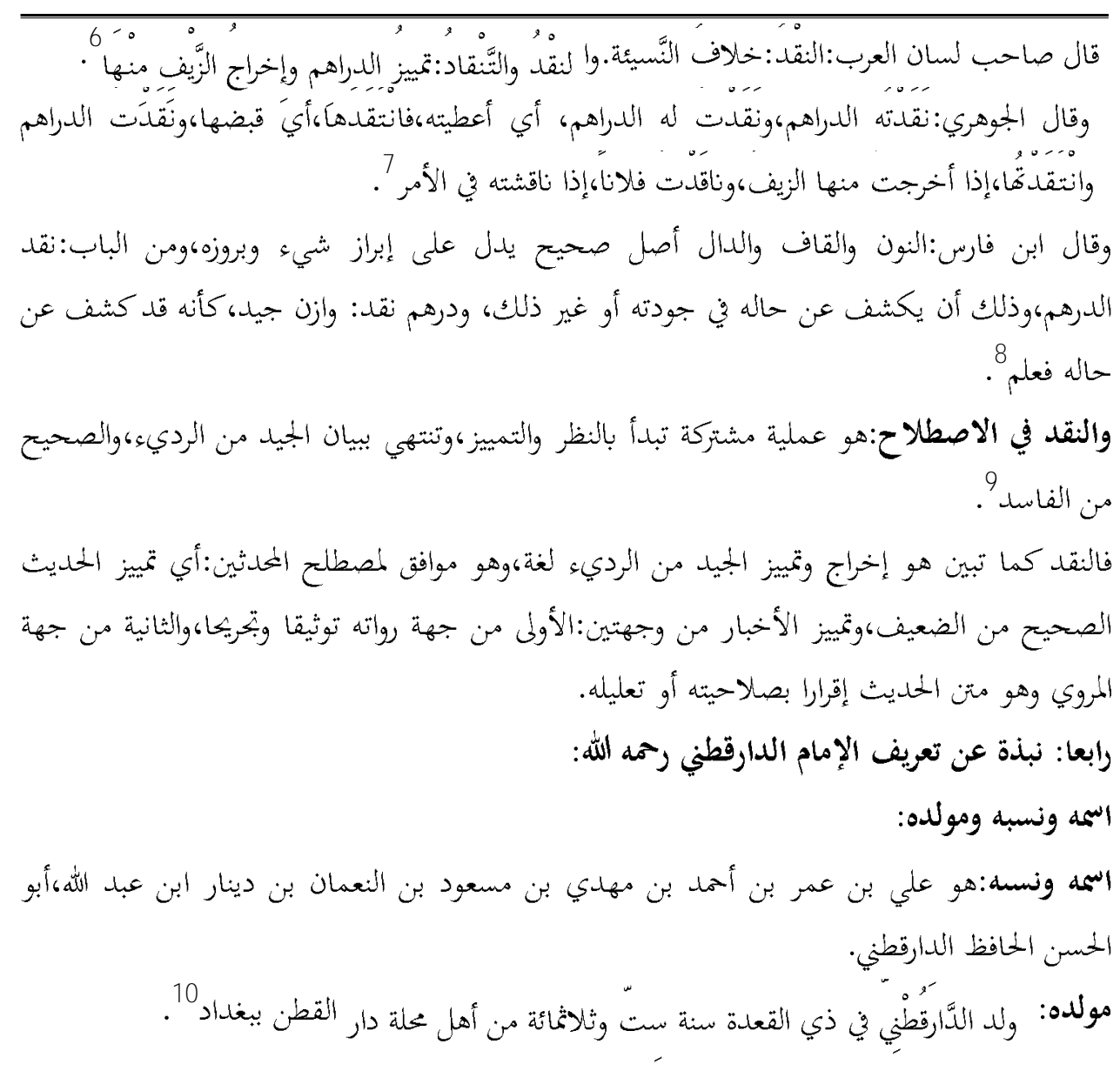

6 _ I be M anzoor, M uhammad, A bu A I-Fazal, Lisan Al-A rab, V.3, P.425, Addition,3,Dar Sadir, Beirut, 1414 A.H .

7 _ Al- Farabi, Ismail I bn H ammad, A/- Sihah Taj Al-A ughah W a Al- Sihah Al-A rabia, V .2,P.544, Addition 4, D ar AI- IIm Lil malaean, Beirut, 1407

A.H.

8 _ Ibe Faris,A hmed, Maqayees Al'lughah, V.5,P.467.

9 _ A bdus Salam Ibn Salih, N aqd A/-Sahabah W at T abean Lit T afseer,V .1, P.16, 1428 A.H

10 _ I bn N uqta, M uhammad Ibn Abdul Ghani, Ikmal A/- I kmal, V.1,P.102, Jamia U mul AI- Q ura, M akka AI- M ukarima, 1410 A.H. 
نشأته وبداية طلبه للعلم:

طلب العلم منذ نعومة أظفاره، واهتم بالحديث رواية ودراية،فبدأ يتردد على مجالس العلماء والمحدثين وعمره لم يتجاوز العشرة. قال أبو الفتح بن أبي الفوارس 11:"كنا نمر إلى البغوي،والدارقطني صبي يكشي خلفنا بيده رغيف عليه "12 12

قال الخطيب البغدادي:"حدثنا الأزهري قال:بلغني أن الدارقطني حضر في حداثثه بجلس إسماعيل

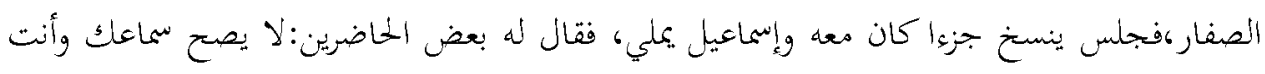

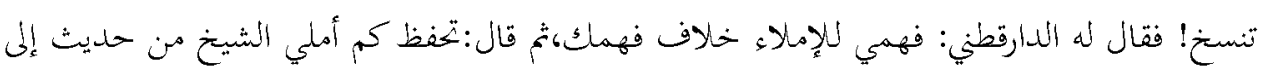

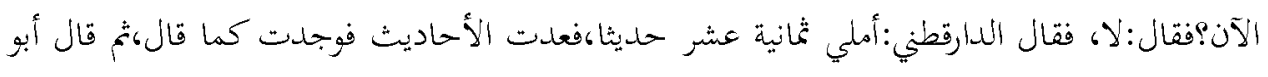

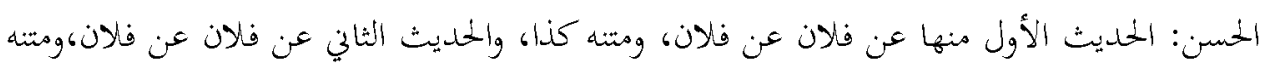

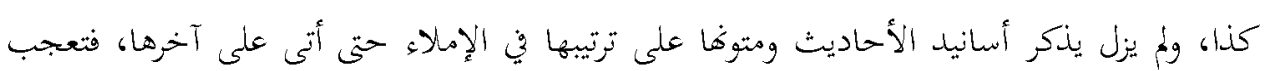
الناس هis 13". ثناء العلماء عليه: الإمام الدارقطني من كبار العلماء في عصره وله معرفة كبيرة في الحمديث وعلومه وعلله وله تصانيف كثيرة

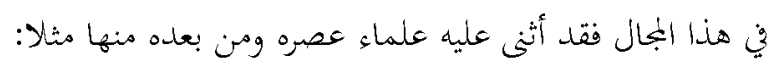

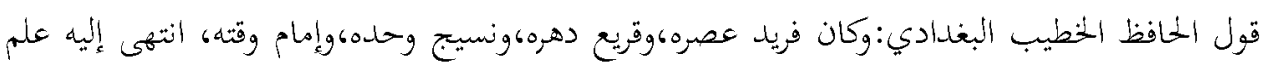

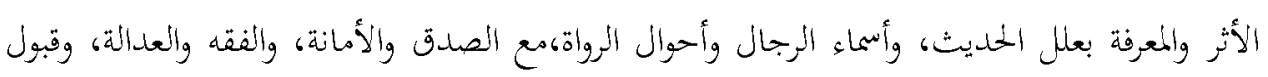
الشهادة،وصحة الاعتقاد، وسلامة المذذهب.

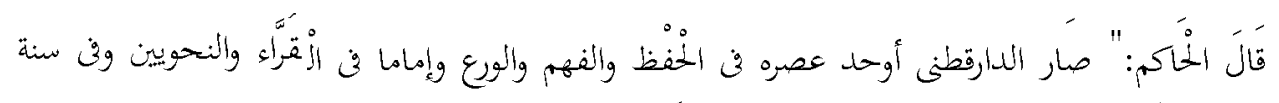

11 _ Az-Z ahabi, M uhamad Ibn A hmad, Sear A alam A/- N ubala, V.16, P.449, Dar Al- H adith, Al- Q ahira, 1427 A.H .

12 Al- Tabrani, Sulaiman Ibn Ahmad, Al- Mu'jam Al-Waseet, V.2, P.798.

13 _ Al-K hateeb Al-Baghdadi, A hmad I bn Ali, Tarikh Baghdád, V .12,P36, D ar Al- K utab Al- IImia, Beirut, 1417 A.H . 


\section{منهجية النقد عند الإمام اللدارقطني في كتابه الأفراد والغرائب}

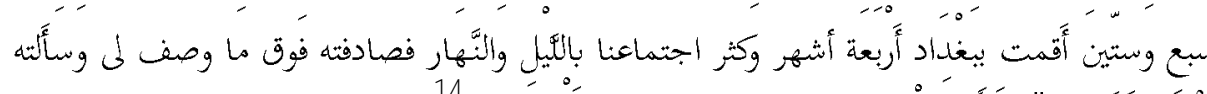

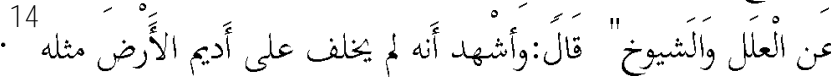

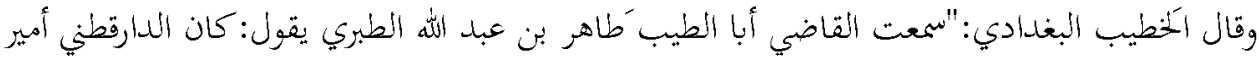
المؤمنين في الحديث، وما رأيت حافظا ورد بغداد إلا مضى إليه،وسلم له 15. 15.".

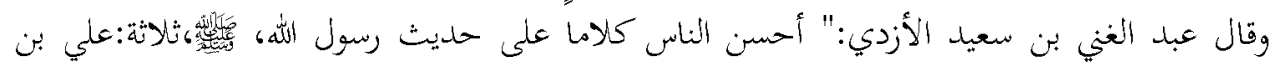

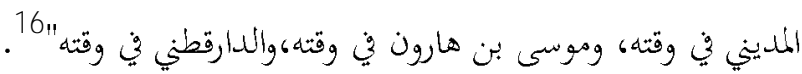

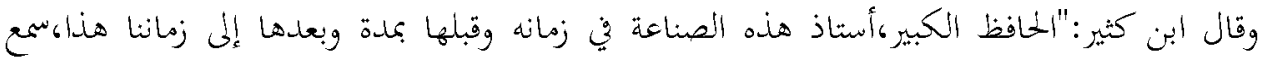

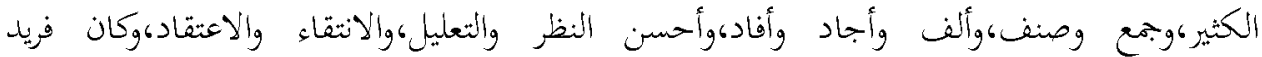

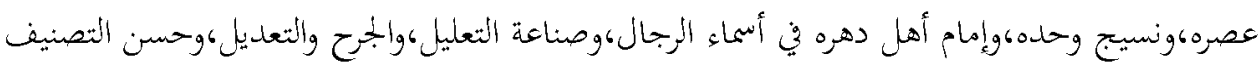

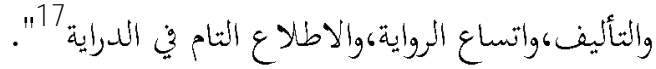

آثاره العلمية: - مانس لقد صنف الإمام الدارقطني في فنون عديدة،منهما في الحديث وعلومه وأسماء الرجال،والقراءات،والفقه، والعقيدة، وكان حسن التصنيف والتأليف،وله مصنفات عديدة منها موجودة ومنها مفقودة،منها

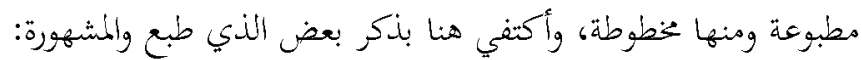

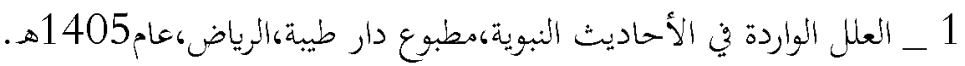

2 _ التبع والإلزامات،طبعا بتحقيق الشيخ مقبل هادي الواعي،الناشر:المكتبة السلفية بالمدينة.

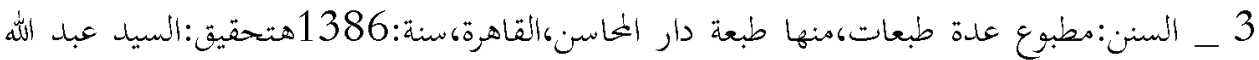

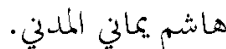
4 _ الأفراد والغرائب،وقةد رتبه الحافظ المقّلدسي فسماه:"أطراف الغرائب والأفراد".

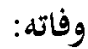

14 _ As-Subki, Abdul W ahab, Tabqat Al-Shafi'ah Al-K ubra, V.3.P.462, Hijr litaba'ati Wa Al- N ashr-e- W a T awzee, 1413 A.H.

15 _ Al-K hateeb A I- Baghdadi, Tareekh Baghdad, V.12,P.35.

16 _ Ibn K halakan, W afyat A/- A 'ayan, V .3,P.298.

17 _ Ibn K athir, Ismail Ibn U mar, A/- Badaya Wa A/-N ahaya, P.15,V .459, Dar Al Fikar, 1407 A.H. 
تويز يوم الأربعاء لثمان خلون من ذي القعدة، وقيل ذي الحجة،سنة خمس وثمانين وثلثمائة

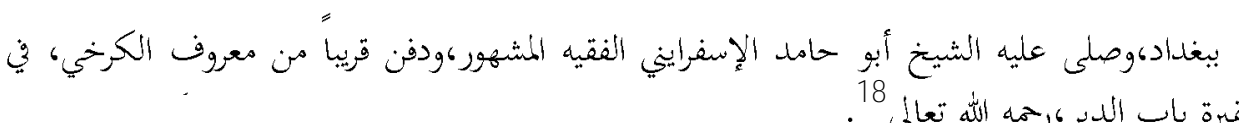

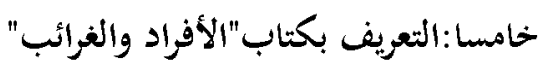

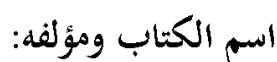
اختلف العلماء في تسمية هذا الكتاب فسماه بعضهم:"الغرائب 19"وسماه بعضهم" الأفرادم"،وسماه

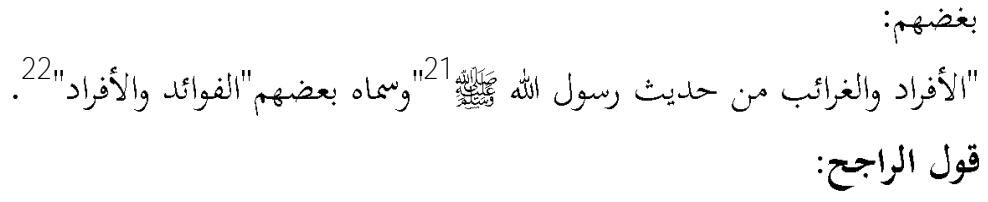
لاشك أن كل اسم من هذه الأسماء هو مصداق حقيقي هذا المكاب العظيم، والحقيقة أن الكتاب

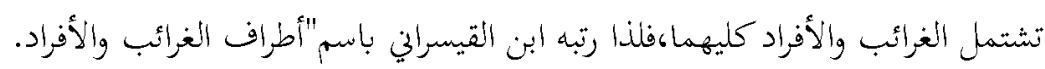

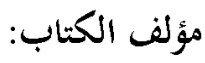
اتفق العلماء والمصنفون أن هذا الكتاب هو تأليف الإمام الدارقطني رحمه الله تعالى. قيمة الكتاب العلمية وعيوبه: الكتاب في غاية الإتقانهودرة ثمينة قلما توجد في هذا الفن، قال الحافظ ابن كثير عن هذا

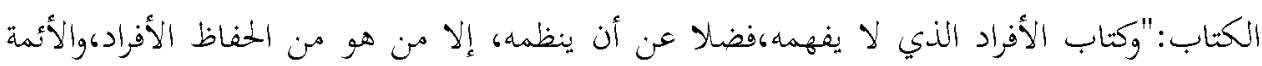

18 _ Ibn K halakan, W afyat A/- A'ayan, V .3,P.298.

19 _ Ibn K halakan, W afyat $A /$ - $A$ 'ayan, V .4,P.287.

20 _ Al- A sqalani, A hmid ibn Ali, Lisan Al- M izan, V.8,P.530, Mua'sasa AlA'alami Lil Matbuat, Beirut, 1390A.H.

21 _ Al-Ishbili, Ibn K hair, Fahrissa, V.1,P.282, D ar AI- G harb A I-Islami Tunisia, 2009 A.D.

22 _ Al- M aharwani, A bo Al-Q asim, Al- $M$ aharwaniat (Al- fawa'id AlM untakhaba T us Sihah W a Al-Ghra'ib),V .1,P.181, Islamic U niversity $M$ adina M unawara. 


\section{منهجية النقد عند الإمام الدارقطني في كتابه الأفراد والغرائب}

الثقاد، والجهابذة الجهياد"23"،وقال أيضا: "وللحافظ الدارقطني كتاب في الإفراد في مائة جزء،ولم يسبق إلى

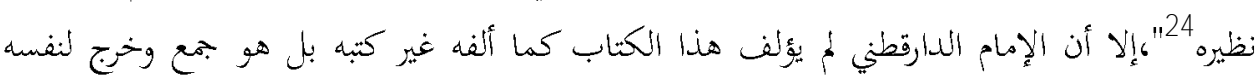

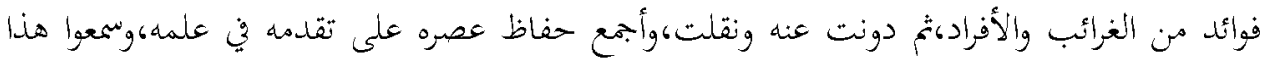

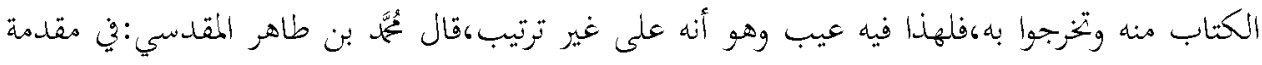

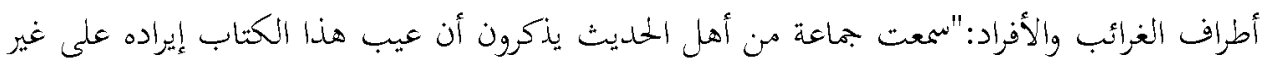

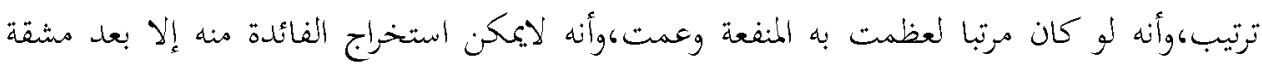

وقال أيضا:عندما دخلت بغداد في رحلتي الأولى عام 467هـ كنت أنتظر شيخي مع مجموعة من طلبة

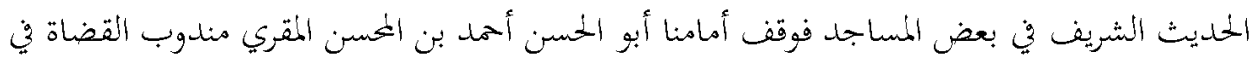

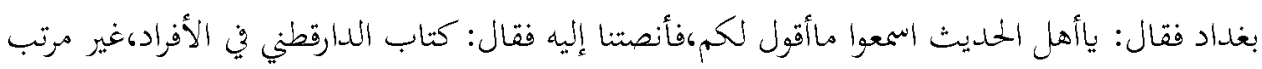

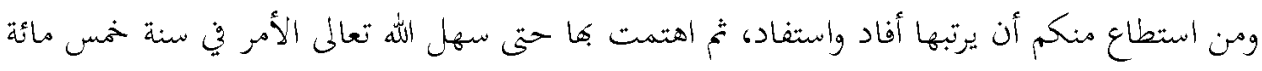

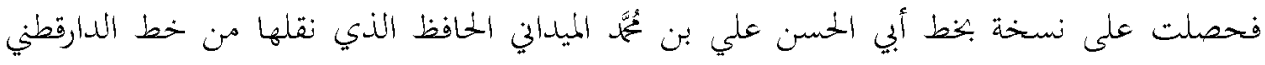

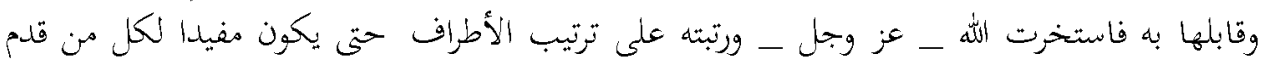

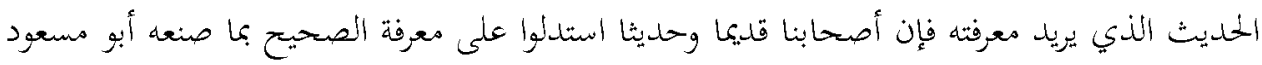

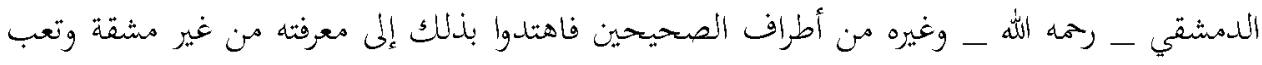

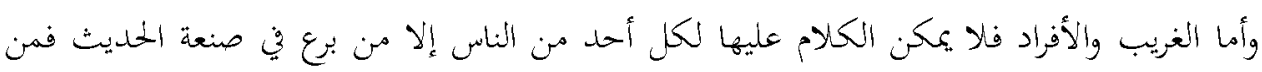
جمع بين هذين الكتابين أمكنه الككلام على أكثر الصحيح والغريب والأفراد.

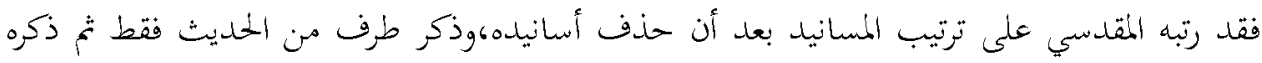
تعليق الإمام الدارقطني عقب الحديث مراعيا بالحروف المعجم في أصحاب المسانيد، فجعله في خمسة فصول،وهي: الإمان الفصل الأول:مسانيد العشرة المُشرين بالجنة فئدئ.

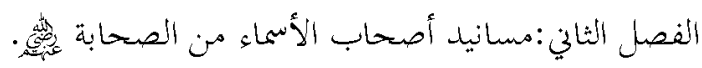
الفصل الثالث:أصحاب الكنى منهم ئئئ.

23 _ Ibn Kathir, Al- Badaya Wa Al-N ahaya,V .11,P362.

24 _Ibn Kathir, Ikhtasar U loom Al- H adith,V .1,P.61. 


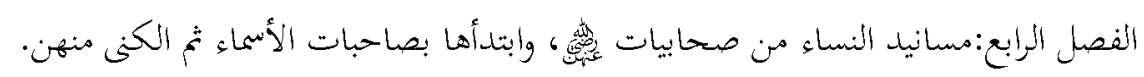

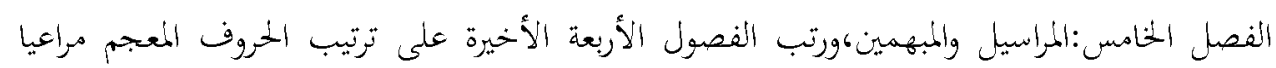

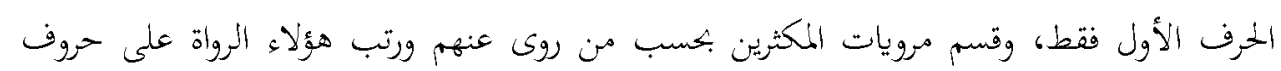

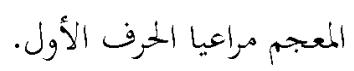

سادسا: منهجية النقد عند الإمام الدارقطني رحمه الله في هذا الكتاب، مع الأمثلة.

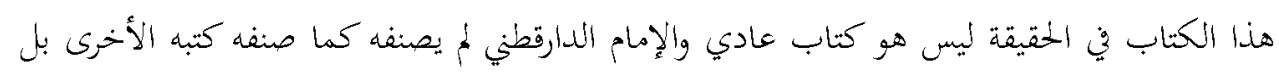

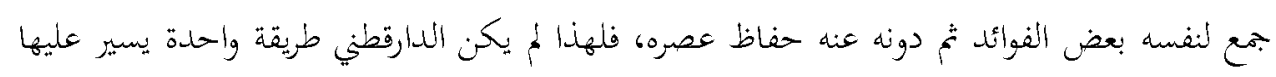

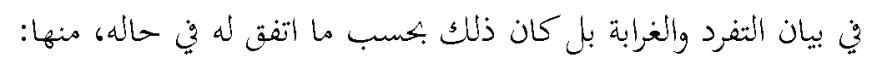

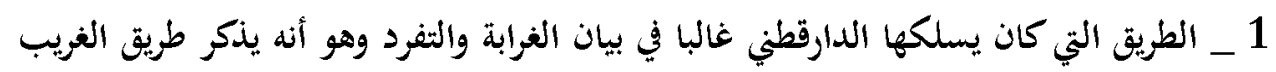

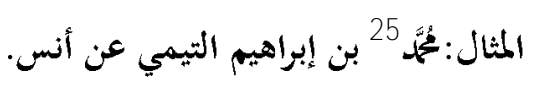

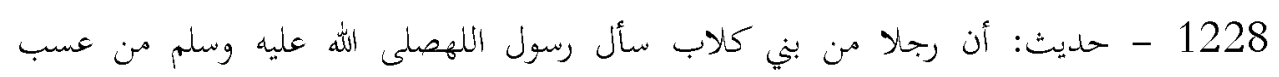

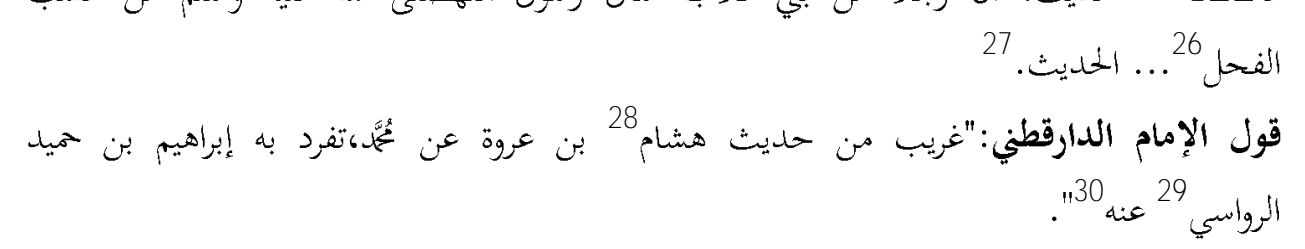

25 - .Al-A sqalani, Ibn H ajar, A hmid ibn Ali, Taqreeb Al- Tahzeeb, P.465, Dar Al- R asheed Syria,1406 A.H .

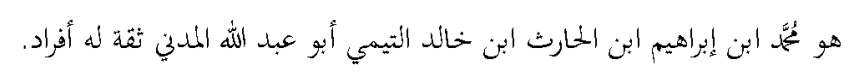

26 - Ibn Al-A sir, AI-M ubarak Ibn M uhammad, Al- N ihaya Fee Ghareeb

Al- hadith Wal'asar, V .3,P234, Al-M aktaba Al'ilmia, Beirut, 1979 A.D.

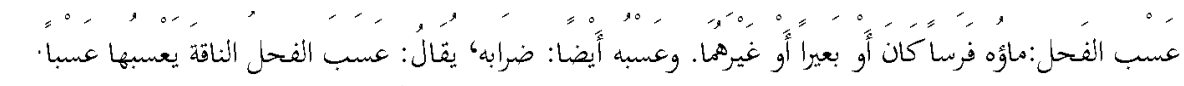

27 - Al- M aqdasi, A traf Al-Ghraib W al A frad,V .2,P. 230.

28 _ Al-A sqalani, Ibn H ajar, Taqreeb A/- T ahzeeb, P.573.

29 _ Al-A sqalani, Ibn H ajar, Taqreeb Al- Tahzeeb, P.89.

هو هشام ابن عروة ابن الزبير ابن العوام الأسدي ثقة فقيه ريكا دلس.

30_ Al- M aqdasi, A traf Al-Ghraib W al A frad,V .2,P.230.

$$
\text { هو إبراهيم ابن حميد ابن عبد الرمن الزؤاسي أبو إسحاق الكوين،ثقة. }
$$




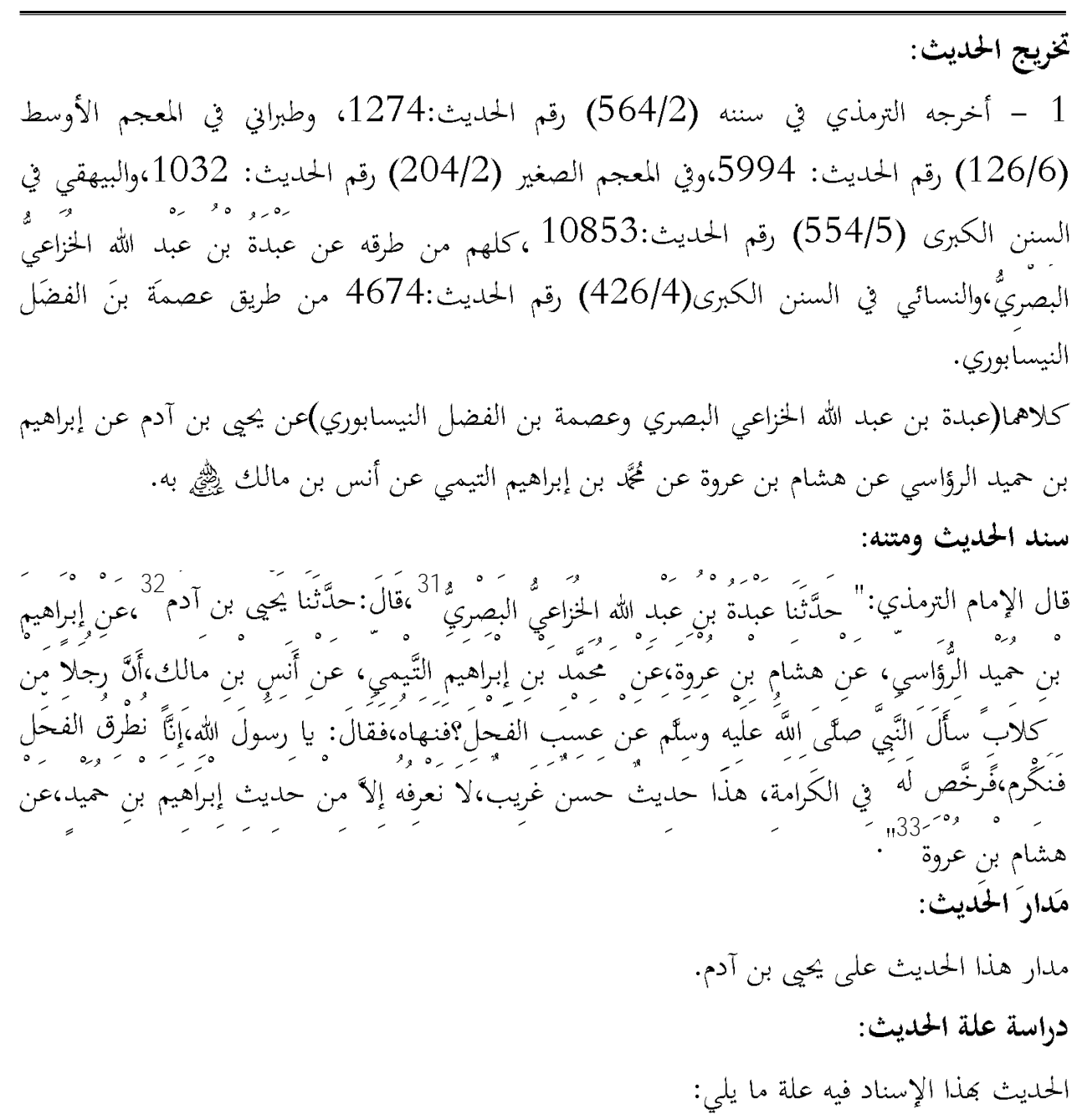

31 _ Al- A sqalani, Ibn H ajar, Taqreeb A/- Tahzeeb, P.369.

هو عبدة ابن عبد الله الصفار الحزاعي أبو سهل البصري كوفي الأصل ثقة. 32_ Al- A sqalani, Ibn H ajar, Taqreeb A/- Tahzeeb, P.587.

هو يميى ابن آدم ابن سليمان الكوين أبو زكريا مولى بني أمية ثثة حافظ فاضل من كبار التاسعة مات سنة ثناث

33 - Al- Tirmazi, Abo E'isa, Muhammad Ibn E'isa, As Sunan, V .2,P564, H adith N 0: 1274, Dar Al- Gharab Al-Islami, Beirut, 1998 A.D. 


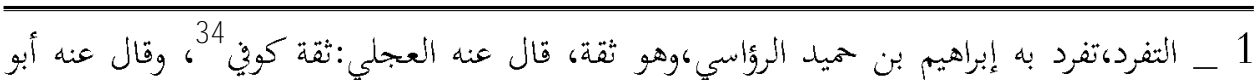

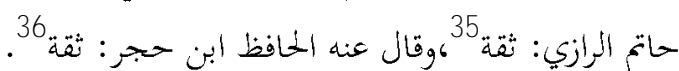

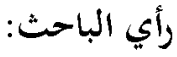

$$
\begin{aligned}
& \text { الحديث بهذا الطريق الذي ساقه الإمام الدارقطني هنا صحيح لأن جميع رواته ثقات مثبتين، قال الإمام } \\
& \text { "هذا حديث حسن لانعرفه إلا من حلديث إبراهيم بن حميد عن هشام بن عروة 37"، وقال الإمام } \\
& \text { الطبراني:"إم يروه عن عُمَّمُ بن إبراهيم إلا هشام بن عروةولا عن هشام إلا إبراهيم بن حميد تفرد به يميى بن }
\end{aligned}
$$

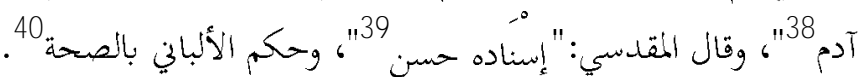

$$
\begin{aligned}
& 2
\end{aligned}
$$

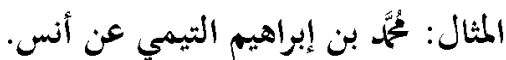

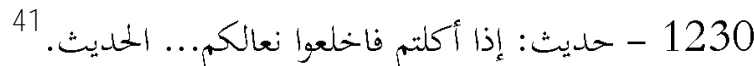

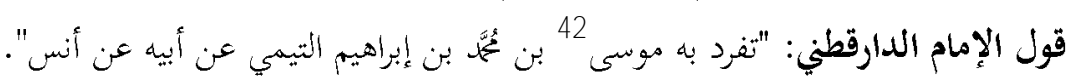

34 _ Al-Ijli, A hmid ibn A bdullah, M arifa T us-Siqat, P.51, M aktaba A I- D ar, $M$ adina M unawara, $1405 \mathrm{~A} . \mathrm{H}$.

35 _ Al-R azi, Abdul R ahman Ibn M uhamad, Al-Jarah Wa Al- Tadeel,

V .2,P249, Dar Ahyah AI-T uras Al-Arbia, Beirut, 1271 A.H .

36 Al-A sqalani, Ibn H ajar, Taqreeb Al- Tahzeeb, P.89.

37- Al- Tirmazi, Abo E'isa, Muhammad Ibn E'isa, As Sunan, V.2,P.564, $\mathrm{H}$ adith N 0: 1274.

38 - Al-T abrani, Sulaiman Ibn A hmid, Al-Mu'jam As-Sagheer,V .2,P204, $\mathrm{H}$ adith $\mathrm{N}$ 0:1032, Al- M aktaba AL_I slami, Beirut, $1405 \mathrm{~A} . \mathrm{H}$.

39 _ Al- M aqasi, M uhammad Ibn Abdul W ahid, A /- A hadith A / M ukhtara, V .7,P.153, H adith N 0:2582, D ar K hizar, Beirut, 1420 A.H.

40_ Al Albani, M uhamad N asir Al- Din, Sahih Wa Za 'ief Sunan al- nasai, V .10,P.230, Hadith N 0: 4672.

${ }^{41}$ _ Al M aqdasi, Atraf Al-Ghraib W a Al-Afrad, V .2,P.230, H adith N o: 1230. 


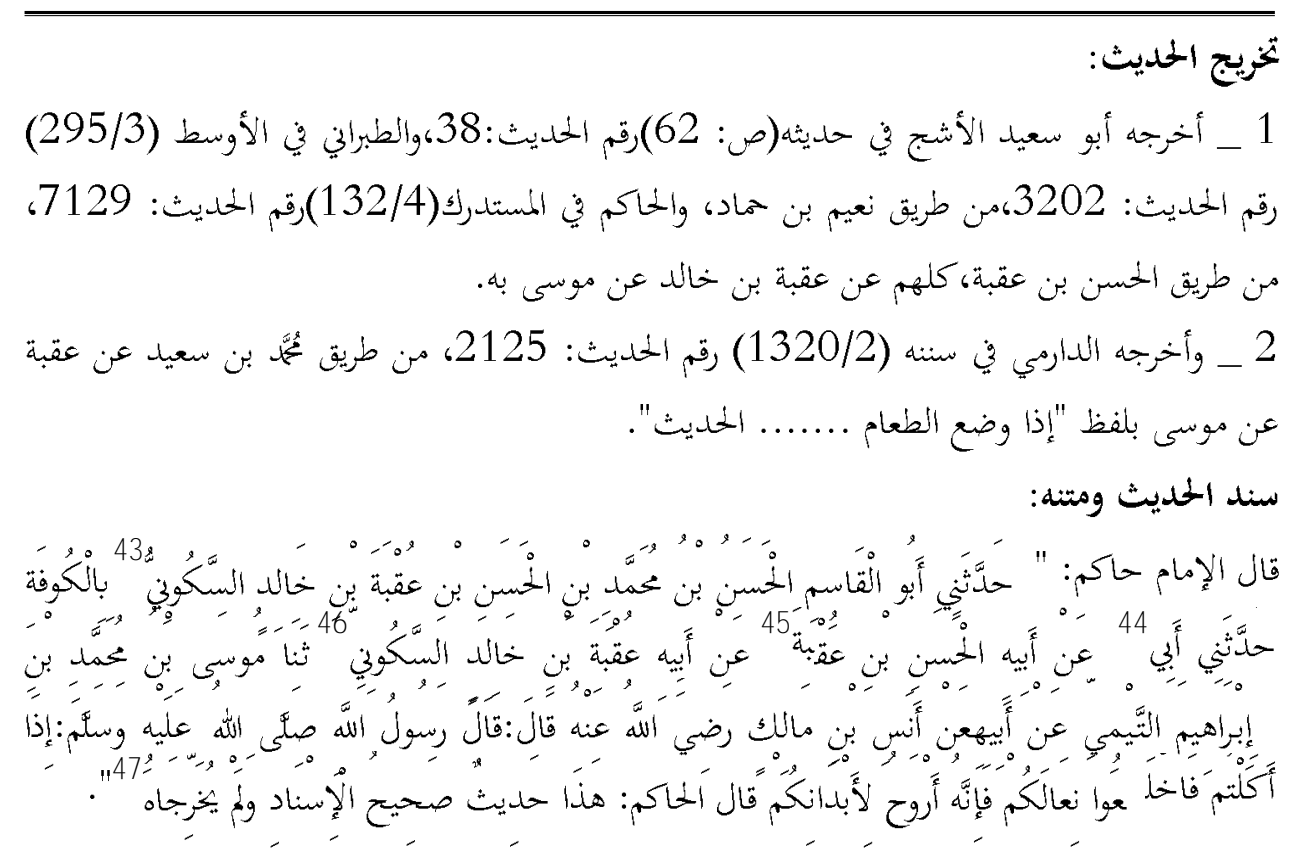

42_ Al- A sqalani, Ibn H ajar, Taqreeb Al- Tahzeeb, P.553.

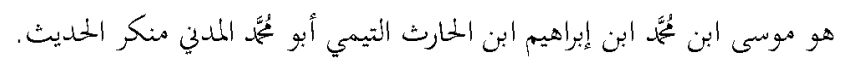

43. Al W adie, M uqbil Ibn H adi, R ijal Al-H akim Fee Al-M ustadrik,

V.1,P.305, M aktaba Sina Al-A tharia, 1415 A.H .

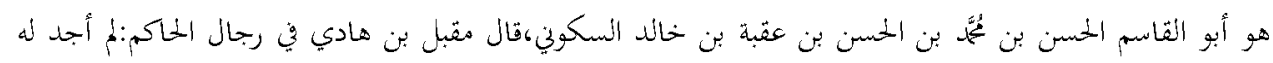
ترجمة.

44 _A I W adie, M uqbil Ibn H adi, R ijal Al-Hakim Fee Al- M ustadrik,

V.2,P.196.

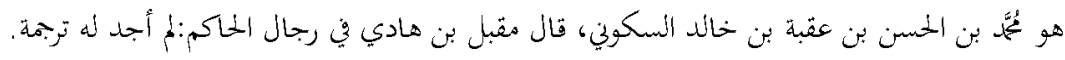

45 _ Al W adie, M uqbil Ibn H adi, R ijal Al- Hakim Fee $A$ I- M ustadrik,

V.1,P.305.

هو الحسن بن عقبة بن خالد السكوين،قال مقبل بن هادي في رجال الحاكم: مُ أجد له ترجمة.

46 _ Al-A sqalani, Ibn H ajar, Taqreeb Al- Tahzeeb, P.394.

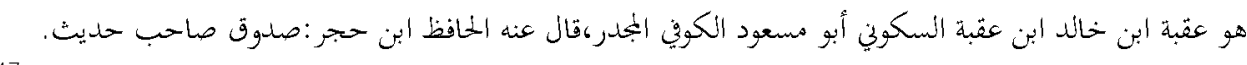

47 _ Al- H akim, M uhammad Ibn Abdullah, Al-M ustadrik Ala Al- Sahehain,

V.4,P.132, H adith N 0: 7129, D ar A I-Kutab AI- IImia, Beirut, 1411 A.H . 


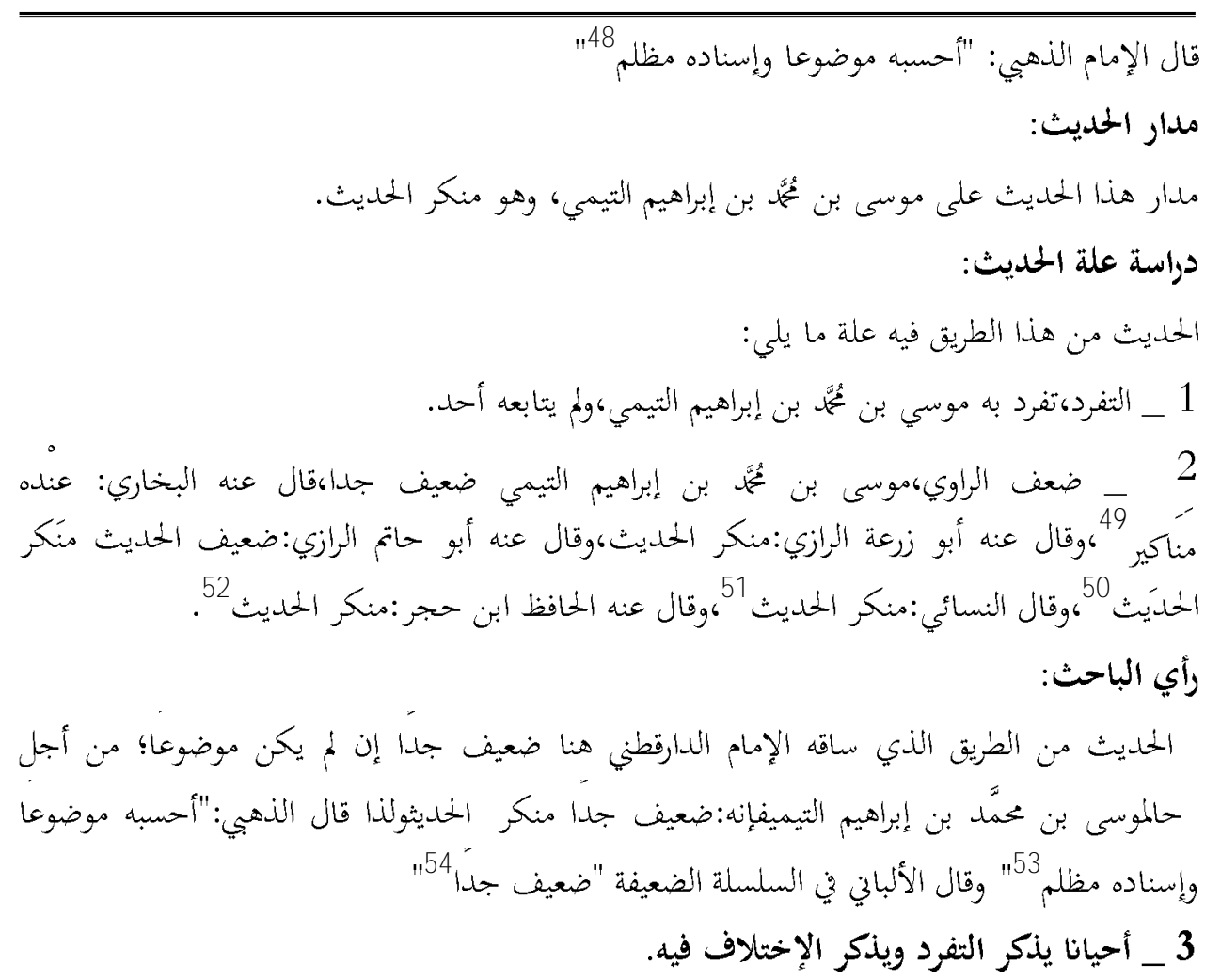

48 _ Al- H akim, M uhammad Ibn Abdullah, Al- M ustadrik Ala Al- Sahehain, V.4,P.132, H adith N 0: 7129.

49 _ Al- Bukhari, M uhammad Ibn Ismail, Al- Tarikh Al- $A$ wsat, V .2,P143,

M aktaba D ar Al-T urath, Al- Q ahira, 1397 A.H .

50 _ Ibn Abi Hatim, Al-Jarh WaAl-Tadil, V .8,P.160.

${ }^{51}$ _ Al- N asai, A hmid Ibn Shoib, Al-Zo'afa Wal Matrokoon, P.95. D ar Al-

Wa'ie, Halb, 1396 A.H.

52 _ Al- A sqalani, Ibn H ajar, Taqreeb A/- Tahzeeb, P.553.

53 - Al- H akim, M uhammad Ibn A bdullah, Al- M ustadrik Ala A/- Sahehain, V.4,P.132, H adith N 0: 7129.

54 _ Al-Albani, M uhammad N asir A I-Din, Silsila A/- Ahadith A/- Zai'efa

Wal Mawzo'ia, V .2,P.411, H adith N 0:980, D ar Al- Mua'rif, Al-R iaz, 1412 A.H. 


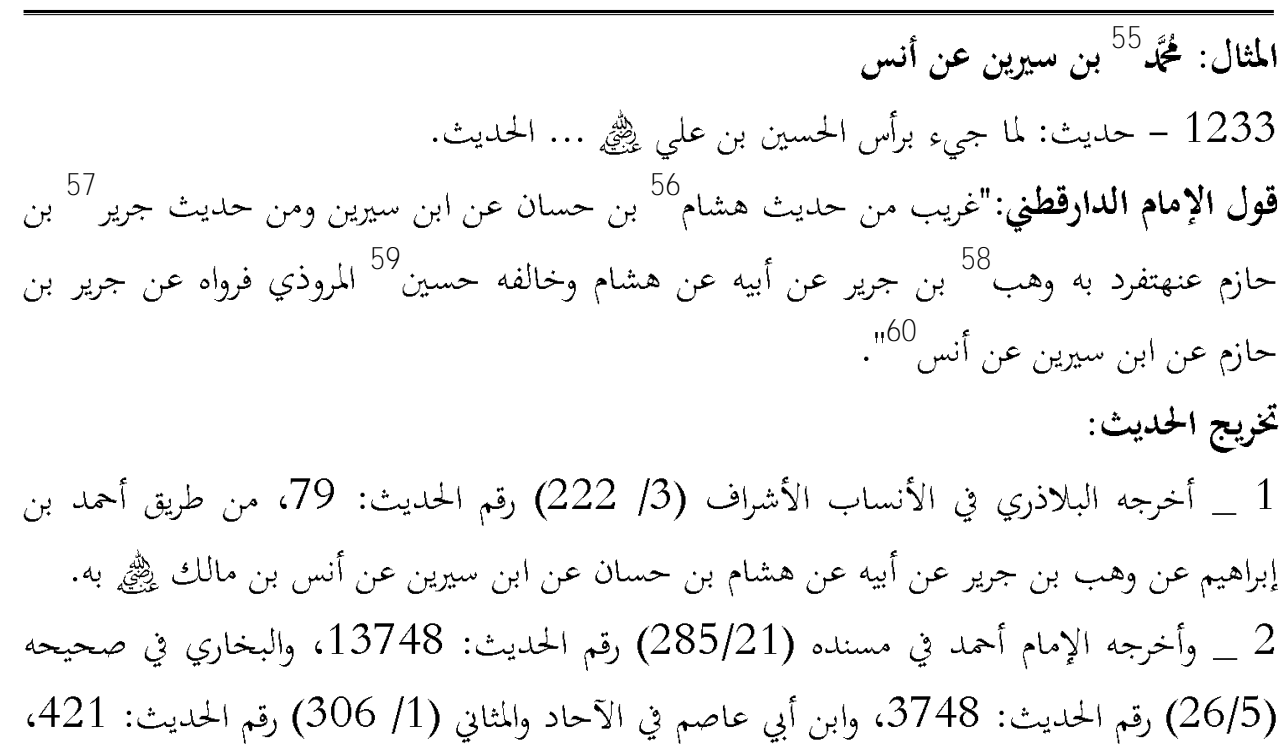

55 _ Al- A sqalani, Ibn H ajar, Taqreeb A/- Tahzeeb, P.483.

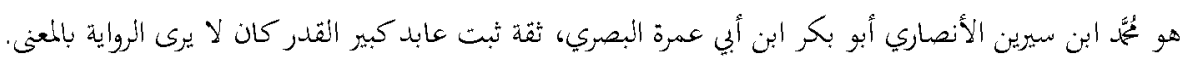

56_ Al- A sqalani, Ibn H ajar, T aqreeb A/- Tahzeeb, P.572.

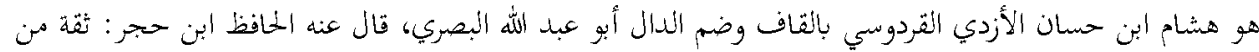

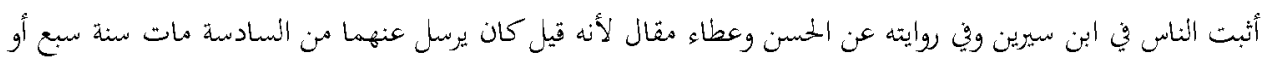

57 _ Al-A sqalani, Ibn H ajar, Taqreeb A/- Tahzeeb, P.138. مثمان وأربعين.

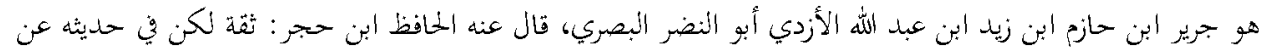

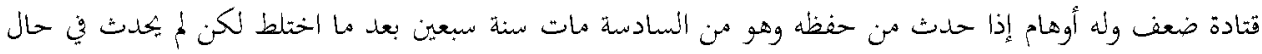

58_ Al-A sqalani, Ibn H ajar, Taqreeb A/- Tahzeeb, P.585.

$.6)$

59 _ Al-A sqalani, Ibn H ajar, Taqreeb Al- Tahzeeb, P.168.

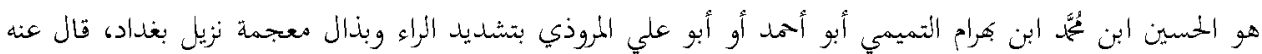

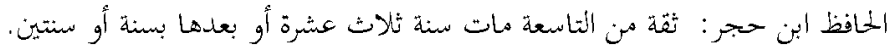

60 _ Al-M aqasi, M uhammad Ibn Abdul W ahid, A traf Al-G Garaib W al Afrad, V .2,P.231, H adith N 0:1233. 


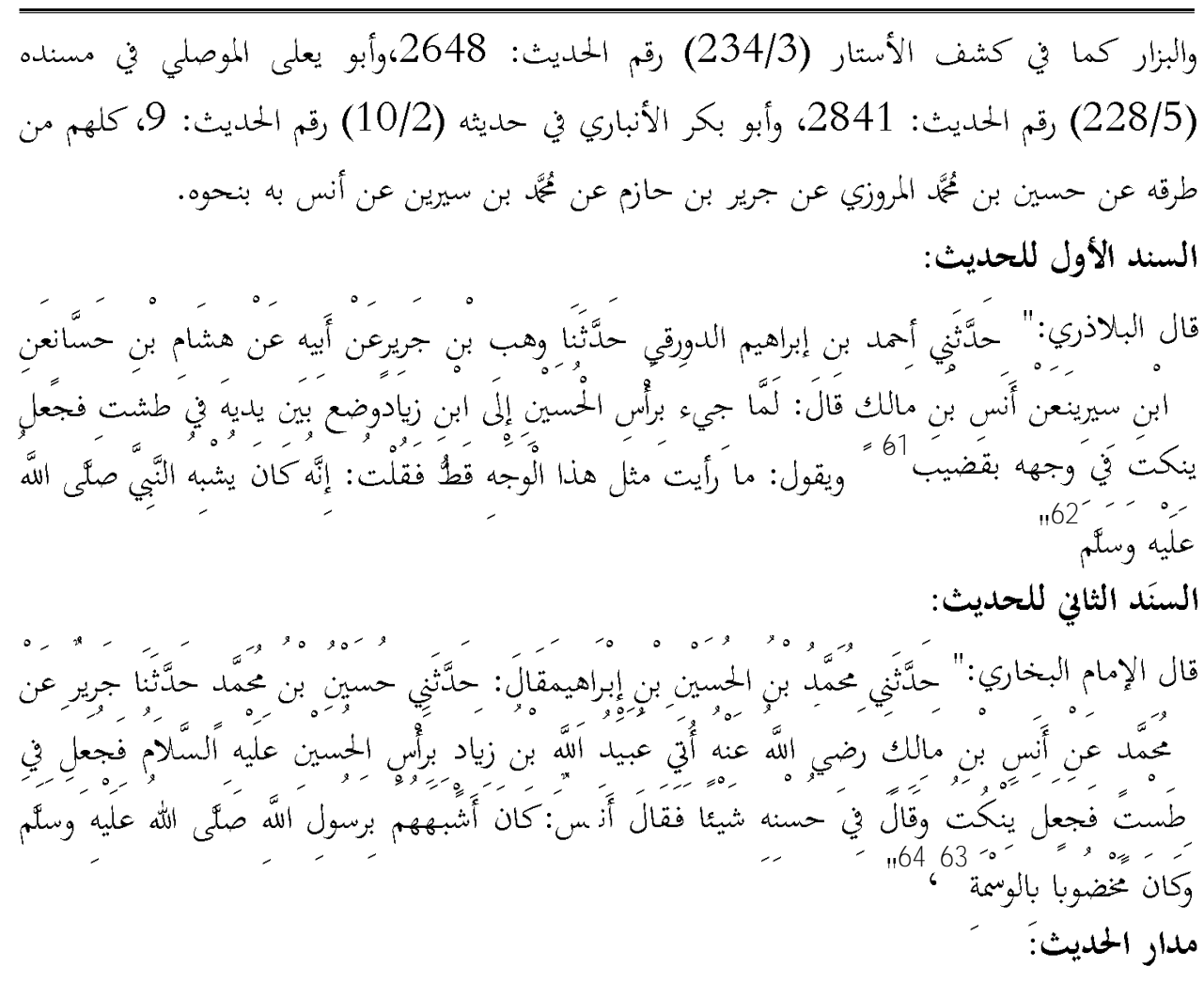

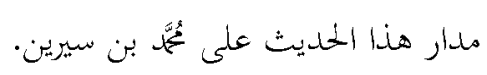

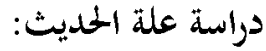

الحديث بهذا الإسناد الذي ساقه الإمام الدارقطني هنا فيه علة ما يلي: 1 ـ التفردكتفرد به وهب بن جرير عن أبيه عن هشام عن ابن سيرين عن أنس ولم يتابعه أحد،وتفرد

61 _Ibn Al-A seer, A/- N ahaya Fee Ghareeb A/- H adith, V .4,P.76.

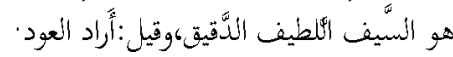

62_ Al-Blazari, A hmid ibn Y ahya, Jumal M in Ansab Al-A shraf, V .3,P.222,

Dar Al- Fikar, Beirut, 1417 A.H .

${ }^{63}$ _ Ibn Al-A seer, Al- N ahaya Fee Ghareeb Al- H adith, V .5,P.185.

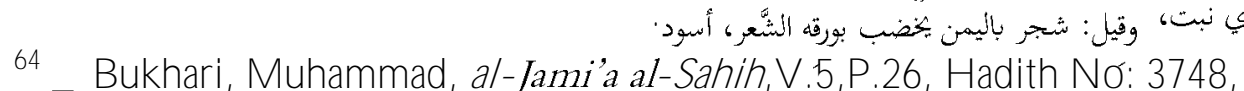
Dar T uraq al- N ajah, 1422 A.H . 


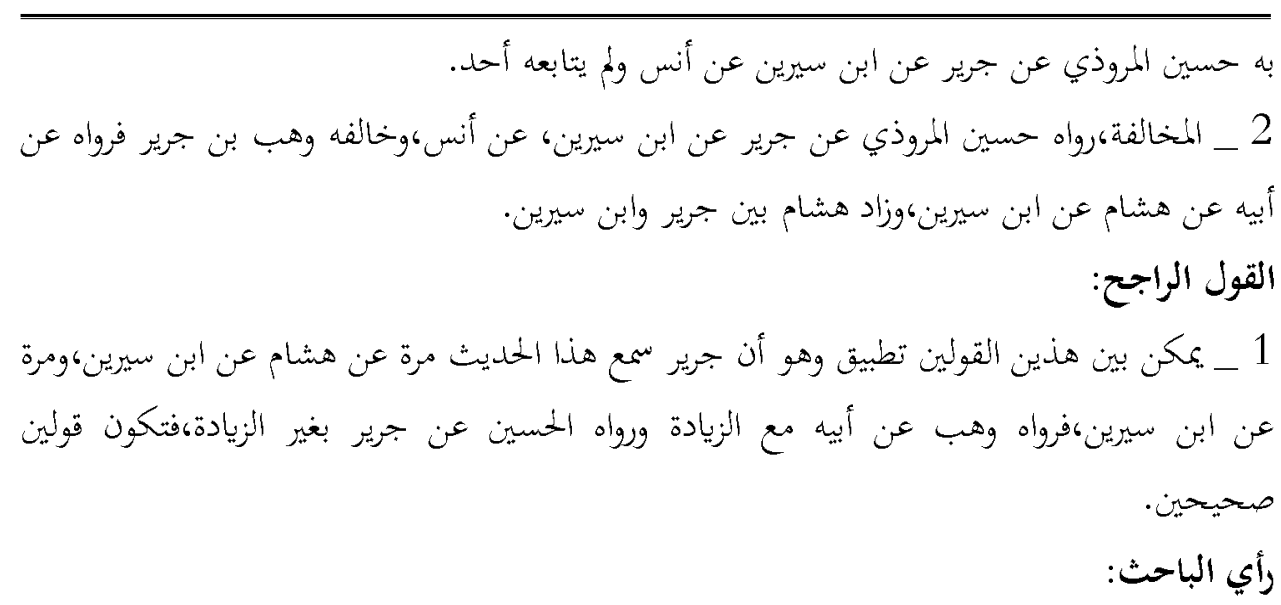

الحديث من الطريق الذي ساقه الإمام الدارقطني هنا فيه اختلاف بين الزيادة ونقصان وكلا الطريقين

4 _ أحيانا يذكر المتن ويذكر الألفاظ التي تفرد به فلان، وأشار إليه.

$$
\text { المثال: موسى بن أنس } 65 \text { عن أبيه }
$$

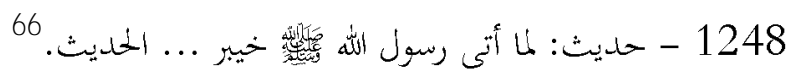

قول الإمام الدارقطني: "وفيه إذا سمع أذاناغريب من حلديث يهيى بن سعيد الأنصاري 67 عن حميد 68 ميد

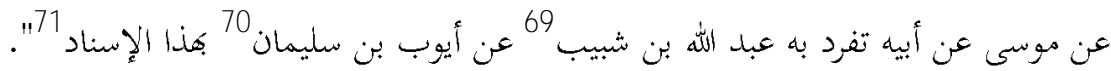

65 Al-A sqalani, Ibn H ajar, Taqreeb Al- Tahzeeb, P.549.

66 Al-M aqasi, M uhammad Ibn A bdul W ahid, A traf A /- Gharaib W al Afrad, V.2,P.237, H adith N 0:1248.

67 _ Al-A sqalani, Ibn H ajar, Taqreeb A/- Tahzeeb, P.591.

68 _ Al- A sqalani, Ibn H ajar, Taqreeb A/- Tahzeeb, P. 181.

$$
\text { هو يجيى ابن سعيد ابن قيس الأنصاري المدني أبو سعيد الثقاضي ثنة ثبت. }
$$

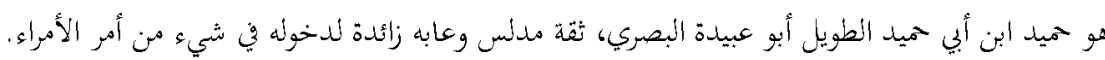

69 _ Sakhavi, M uhammad, Al- T uhfa T ul Latifa Fee Tarikh Al-M adina A/-

Sharifa, V .2,P.42, Al- Kutab Al- IImia, Beirut,1414 A.H .

هو عبد الله بن شبيب بن خالد: أبو سعيد الربعي مولاهم المدني الأخباري العلامة من أهل البصرة، قال الحاكم: هو

70 Al- A sqalani, Ibn H ajar, Taqreeb A/- Tahzeeb, P.118.

$$
\text { ذاهب الحمديث. }
$$




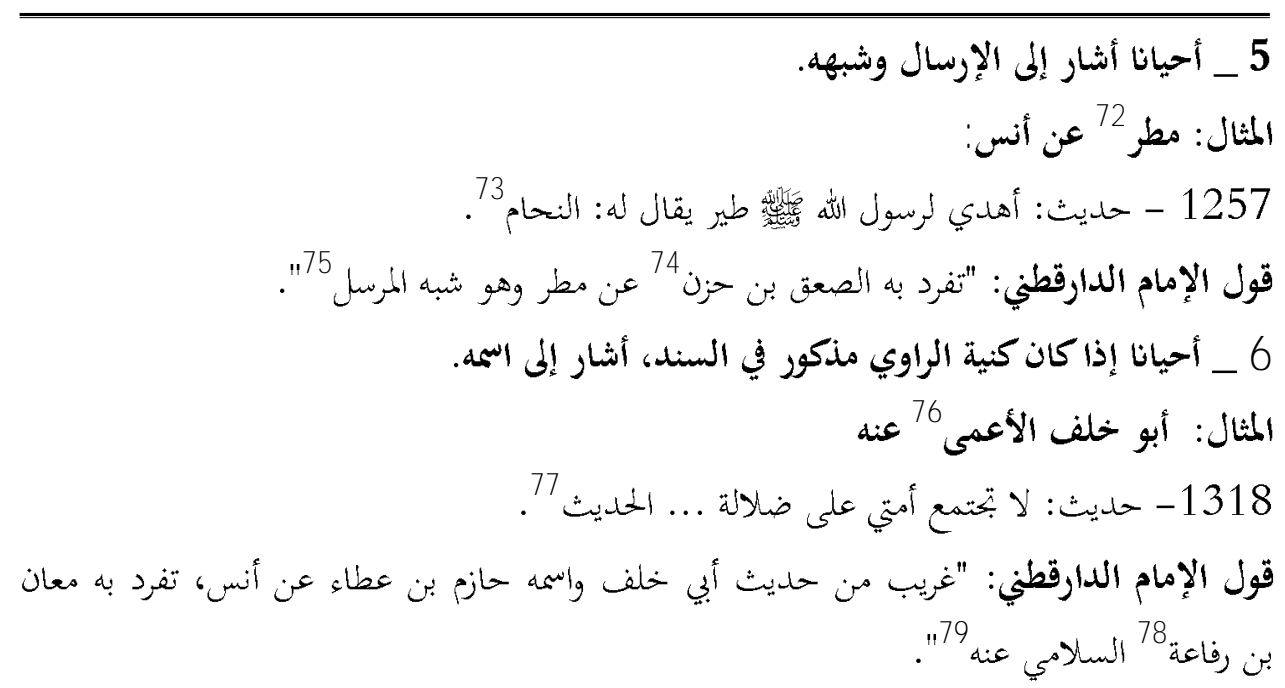

هو أيوب ابن سليمان ابن بلال المدني أبو يجيى ثقة لينه الأزدي وانساجي بلا دليل.

71 _ Al-M aqasi, M uhammad Ibn Abdul W ahid, Atraf A/-Gharaib Wal A frad, V.2,P.237, H adith N 0:1248.

72 _ Al- A sqalani, Ibn H ajar, Taqreeb A/- Tahzeeb, P.534.

هو مطر ابن طهمان الوراق أبو رجاء السلمي مولاهم الحزراساني سكن البصرة صدوق كثير الخطأ وحديثه عن عطاء ضعيف من السادسة مات سنة همس وعشرين ويقال سنة تسع.

73 _ Al- M aqasi, M uhammad Ibn Abdul W ahid, A traf Al-G Garaib W al Afrad, V.2,P.240, H adith N 0:1257.

74_ Al-A sqalani, Ibn H ajar, Taqreeb A/- T ahzeeb, P.276.

75 _ Al-M aqasi, M uhammad Ibn Abdul W ahid, A traf Al-G Garaib W al Afrad, V.2,P.240, H adith N 0:1257.

76 _ Al-A sqalani, Ibn H ajar, Tahzeeb A/- Tahzeeb,V .12, P.87.

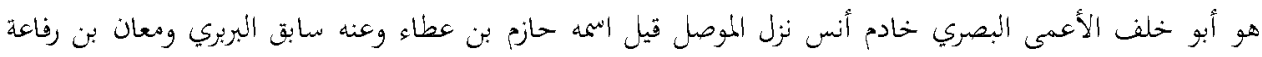

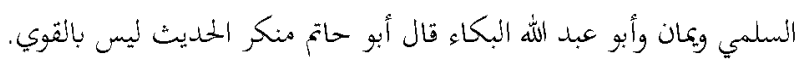

77 _ Al- M aqasi, M uhammad Ibn Abdul W ahid, A traf Al- Gharaib W al Afrad, V.2,P.261, H adith N 0:1318.

78 _ Al- A sqalani, Ibn H ajar, Tahzeeb A/- Tahzeeb, P.537.

هو معان ابن رفاءة السلامي الشامي لنين الحديث كثير الإرسال. 


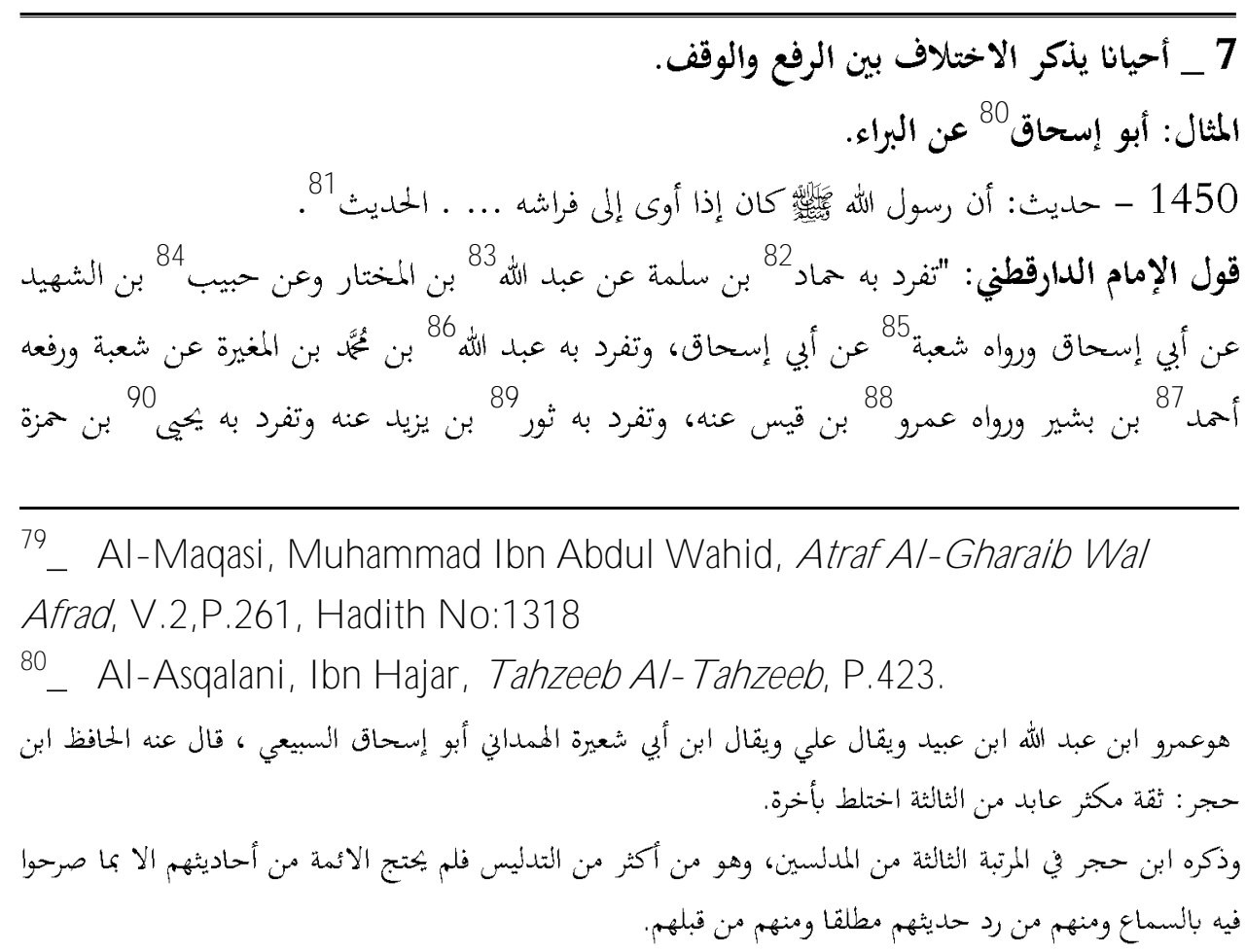

Al- A sqalani, Ibn H ajar, Tabqat A I- M udalliseen, V .2,P.310, M aktaba Al-

M annar, U mman,1403 A.H .

81 _ Al-M aqasi, M uhammad Ibn A bdul W ahid, A traf Al- Gharaib W al Afrad, V.2,P.310, H adith N 0:1450.

82_ Al- A sqalani, Ibn H ajar, Taqreeb A/- Tahzeeb, P.178.

هو حماد ابن سلمة ابن دينار البصري أبو سلمة ثقه عابد أثبت الناس في ثابت وتغير حفظه بأخرة.

83 _ Al- A sqalani, Ibn H ajar Taqreeb Al- Tahzeeb, P.322.

84 _ Al- A sqalani, Ibn H ajar, Taqreeb Al- Tahzeeb, P.151.

هوعبد الله ابن المختار البصري، لابأس به.

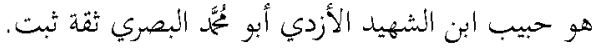

85 _ Al- A sqalani, Ibn H ajar, Taqreeb Al- Tahzeeb, P.266.

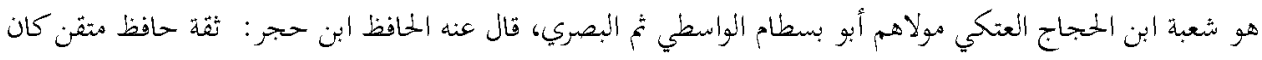

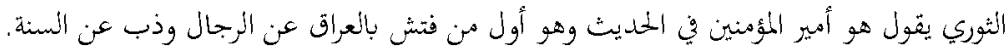

86 _ Ibe Abi H atim, Abdul R ahman, Al-Jarh wa al- Tadeel, V.5,P.158.

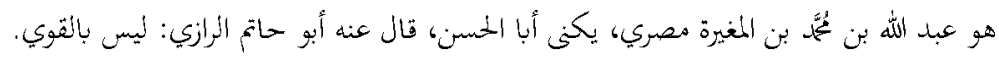

87 _ Ibe Abi H atim, Abdul R ahman, Al-Jarh wa al- Tadeel, V .2,P.42. 


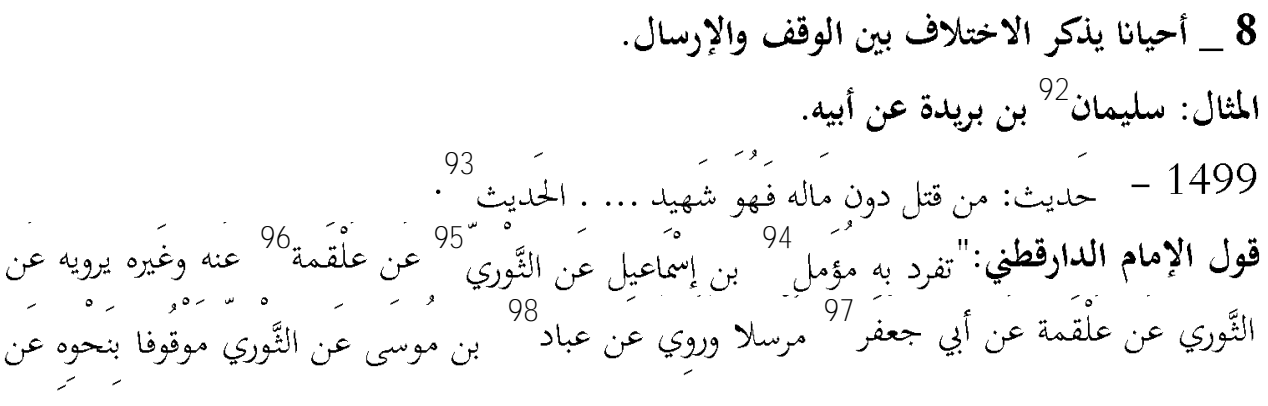

$$
\text { هو أحمد بن بشير أبو بكر مولى عمرو بن حريث المخزومي، قال عنه أبو زرعة الزازي: صدوق. }
$$

88 _ Al-A sqalani, Ibn H ajar T aqreeb A/- Tahzeeb, P.436.

89_Al-A sqalani, Ibn H ajar, Taqreeb Al- Tahzeeb, P.135.

هو عمرو ابن قيس الملائي أبو عبد الله الكوفي، ثقة متقن عابد.

90 _ Al-A sqalani, Ibn H ajar, Taqreeb A/- Tahzeeb, P.589.

$$
\text { هو ثور ابن يزيد الحمصي، ثثة ثبت إلا أنه يرى القدر. }
$$

هو يميى ابن حمزة ابن واقد الخضرمي أبو عبد الرمن، ثقة رمي بالقدر.

91 _ Al-M aqasi, M uhammad Ibn Abdul W ahid, A traf Al-G Garaib Wal Afrad, V.2,P.310, H adith N 0:1450.

92 _ Al- A sqalani, Ibn H ajar, Taqreeb A/- T ahzeeb, P.250.

هو سليمان ابن بريدة ابن الحصيب الأسلمي المروزي قاضيها تثة.

93 _ Al-M aqasi, M uhammad Ibn Abdul W ahid, A traf Al- Gharaib W al A frad, V.2,P.324, Hadith N 0:1499.

94_ Al-A sqalani, Ibn H ajar, Taqreeb A/- Tahzeeb, P.555.

95 _ Al-A sqalani, Ibn H ajar, Taqreeb A/- Tahzeeb, P. 244.

$$
\text { هو مؤمل بن انماعيل أبو عبد الرمن، صدوق سيئ الحفظ. }
$$

هو سفيان ابن سعيد الثوري أبو عبد الله الكوين، ثقة حافظ نقيه عابد إمام حجة.

96 _ Al- A sqalani, Ibn H ajar, Taqreeb Al- Tahzeeb, P.397.

97 _ Al- A sqalani, Ibn H ajar Taqreeb A /- Tahzeeb, P.497.

$$
\text { هو علقمة ابن مرثد الخضرمي أبو الحارث الكويف، ثقة. }
$$

98 _ Al- A sqalani, Ibn H ajar T aqreeb A /- T ahzeeb, P.291.

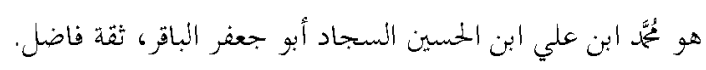$$
\text { هوعباد ابن موسى القرشي أبو عقبة البصري العباداني نزيل بغداد، ثقة. }
$$ 


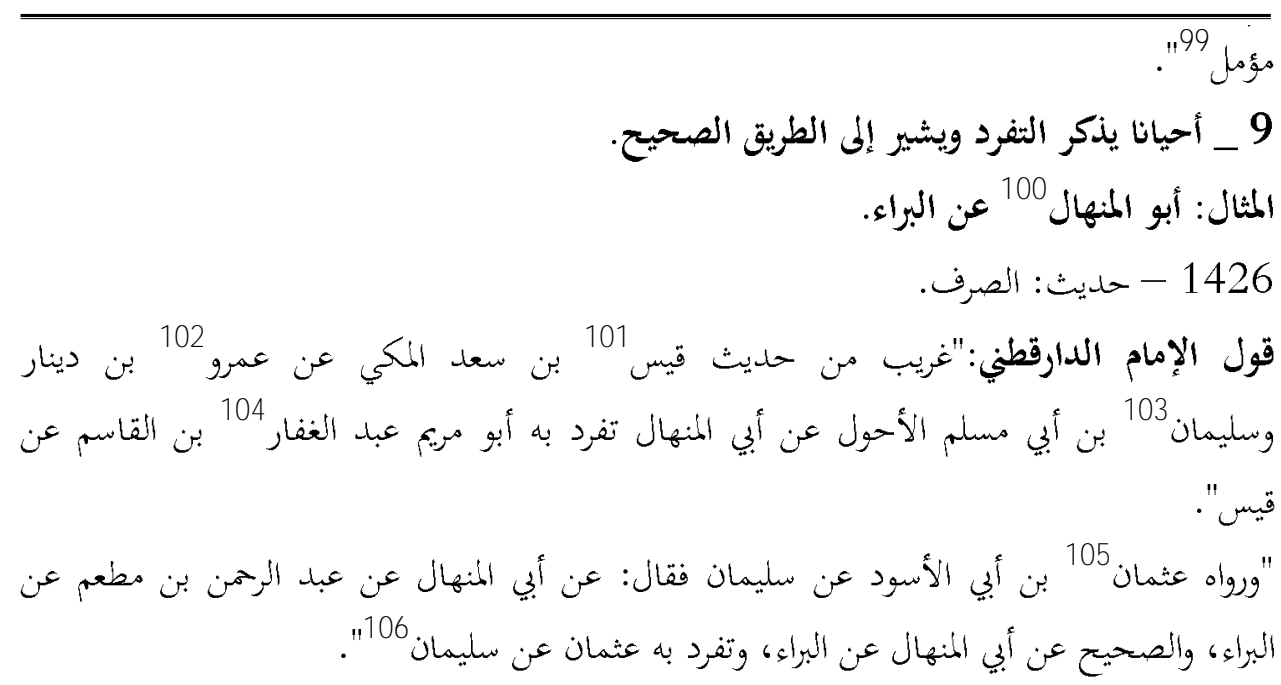

99 _ Al- M aqasi, M uhammad Ibn A bdul W ahid, A traf $A$ /- Gharaib W al A frad, V.2,P.324, H adith N 0:1499.

100 _ Al-A sqalani, Ibn H ajar, Taqreeb A/- Tahzeeb, P.350.

101 _ Al-A sqalani, Ibn H ajar, Taqreeb Al- Tahzeeb, P. 457. هوعبد الرمن ابن مطعم البناني أبو المنهال البصري نزل مكة، ثقة.

102 _ Al- A sqalani, Ibn H ajar, Taqreeb Al- T ahzeeb, P.421. هو قيس ابن سعد المكي، تثة.

103 _ Al- A sqalani, Ibn H ajar, Taqreeb Al- T ahzeeb, P. 254.

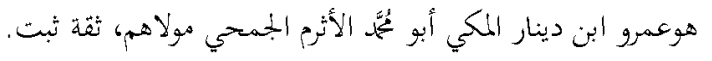

هوسليمان ابن أبي مسلم المكي الأحول قيل اسم أبيه عبد الله، ثقة ثنقة. 104 _ Baghdadi, Yahya Ibn Mui'en, Tareekh Ibn Moi'en,V.3,P.366, A hya Al- T urath Al-I slami, M akka AL- M ukarma, 1399 A.H .

هوعبد الغفار بن القاسم أبو مريم الغفاري كوف وهو ابن القاسم بن قيس بن قهد ابن عم يحيى بن سعيد الأنصاري، قال

105 _ Al-A sqalani, Ibn H ajar, Taqreeb Al- T ahzeeb, P.382. عنه ابن معين: ليس بشيئ. هوعثمان ابن الأسود ابن موسى المكي مولى بني جمح، ثقة ثبت. 106 _ Al- M aqasi, M uhammad Ibn A bdul W ahid, A traf Al- Gharaib W al A frad, V.2,P.303, H adith N 0:1426. 


$$
\begin{aligned}
& 10 \text { _ أحيانا يذكر الثفرد حسب علمه. }
\end{aligned}
$$

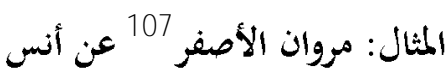

$$
\begin{aligned}
& 1265 \text { - حديث: ما قدم علي من اليمن ... . . الحديث. } 108
\end{aligned}
$$

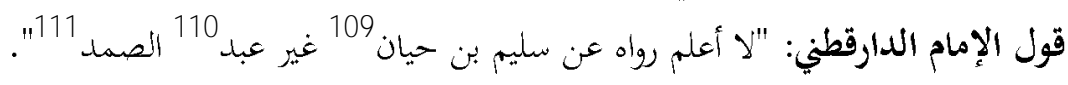

$$
\begin{aligned}
& 11 \text { _ أحيانا يذكر الغرابة فتط ولم يذكر من تفرد عنه. } \\
& \text { المثال : يجيى بن سعيد الأنصاري عن أنس }
\end{aligned}
$$

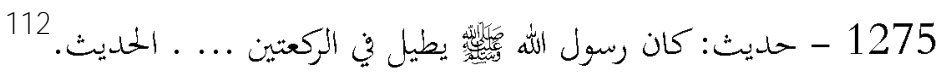

$$
\begin{aligned}
& \text { قول الإمام الدارقطني: غريب من حديث يميى الأنصاري عن أنس. } \\
& 12 \text { _ أحيانا يذكر التفرد ويذكر بعده الطريقة الخفوظة. } \\
& \text { المثال: عدي } 113 \text { بن ثابت عن البراء البرد ويدكر بعدو }
\end{aligned}
$$

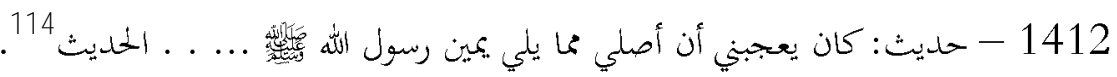

107 _ Al- A sqalani, Ibn H ajar Taqreeb Al- Tahzeeb, P.526.

$$
\text { هو مروان الأصفر أبو خليفة [خلف] البصري قيل اسم أبيه خاقان وقيل سالم ثقة. }
$$

108 _ Al- M aqasi, M uhammad Ibn Abdul W ahid, A traf Al-Gharaib W al

Afrad, V.2,P.244, H adith N 0:1265.

109 _ Al-A sqalani, Ibn H ajar, Taqreeb A/- Tahzeeb, P. 249.

110 _ Al-A sqalani, Ibn H ajar, Taqreeb A/- T ahzeeb, P.356.

$$
\text { هو سليم بفتح أوله ابن حيان بمهملة وثتانية الهذلي البصري ثقة من السابعة. }
$$

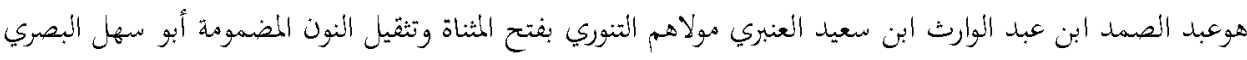
صدوق ثبت في شعبة من التاسعة مات سنة سبع وماثتين.

111 _ Al- M aqasi, M uhammad Ibn Abdul W ahid, A traf Al-G Garaib W al

Afrad, V.2,P.244, H adith N 0:1265.

112 _ Al-M aqasi, M uhammad Ibn Abdul Wahid, Atraf Al-Gharaib Wal Afrad, V.2,P.248, H adith N 0:1275.

113 _ Al-A sqalani, Ibn H ajar Taqreeb Al- Tahzeeb, P.388.

$$
\text { هوعدي ابن ثابت الأنصاري الكوفي، ثقة رمي بالتشيع. }
$$




$$
\begin{aligned}
& \text { قول الإمام الدارقطني:"تفرد به ابن عيينة } 115 \text { عن مسعر } 116 \text { عن عدي وتفرد به عبد الززاق } 117 \text { عن ابن } \\
& \text { عيينة والمحفوظ عن مسعر عن ثابت } 118 \text { بن عبيد عن ابن البراء } 119 \text { عن البراء 120". } \\
& 13 \text { ـ غالبا يذكر التفرد والغرابة ولم يذكر التفصيل ولكن أحيانا يذكر التفصيل والاختلاف فيه. } \\
& \text { المثال: عبد الرحمن بن أبي ليلى عن بلال }
\end{aligned}
$$

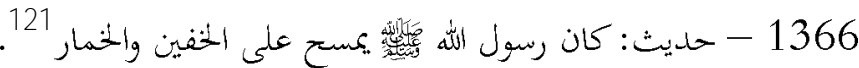

$$
\begin{aligned}
& \text { قال الإمام الدارقطني:"تفرد به سفيان بن عيينة عن أبان بن تغلب } 122 \text { وعبيد الله بن عمرو } 123 \text { عن زيد }
\end{aligned}
$$

114 _ Al- M aqasi, M uhammad Ibn A bdul W ahid, A traf Al- Gharaib W al

Afrad, V.2,P.298, H adith N 0:1412.

115 _ Al-A sqalani, Ibn H ajar, Taqreeb Al- Tahzeeb, P.245.

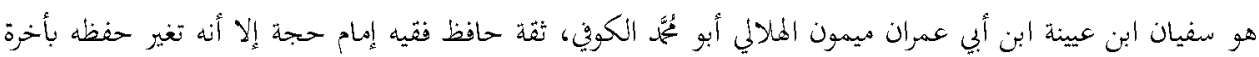

116 _ Al- A sqalani, Ibn H ajar, Taqreeb A/- T ahzeeb, P.528.

وكان ريما دلس لكن عن الثقات.

$$
\text { هو مسعر ابن كدام ابن ظهير الهلالي أبو سلمة الكويف، ثقة تبت فاضل. }
$$

117 _ Al- A sqalani, Ibn H ajar Taqreeb Al- Tahzeeb, P.354.

118 _ Al- A sqalani, Ibn H ajar, Taqreeb Al- Tahzeeb, P.132.

$$
\text { هو عبد الرزاق ابن همام ابن نافع الحميري ثقة حافظ. }
$$

هو ثابت ابن عبيد الأنصاري مولى زيد ابن ثابت كوفي، ثنقة.

119 _ Al-A sqalani, Ibn H ajar, Taqreeb Al- Tahzeeb, P.376.

$$
\text { هو عبيد ابن البراء ابن عازب الأنصاري الحارثي الكوين، ثقة. }
$$

120 _ Al- M aqasi, M uhammad Ibn Abdul W ahid, A traf Al-Gharaib W al Afrad, V.2,P.298, H adith N 0:1412.

121 _ Al-M aqasi, M uhammad Ibn Abdul Wahid, A traf Al-Gharaib Wal Afrad, V.2,P.278, H adith N 0:1366.

122 _ Al- A sqalani, Ibn H ajar, T ahzeeb Al-T ahzeeb, P.87.

123 _ Al- A sqalani, Ibn H ajar Taqreeb Al- Tahzeeb, P.373.

$$
\text { هو أبان ابن تغلب أبو سعد الكوين، ثقة تكلم فيه لنتشيع. }
$$

هو عبيد الله ابن عمرو ابن أبي الوليد الرقي، ثقة فقيه ربما وهم. 
The Scholar Islamic Academic Research Journal

Vol. 7, No. 2 || July-December 2020||P.1-28

https://doi.org/10.29370/siarj/ issue13arabic1

\begin{tabular}{|c|}
\hline 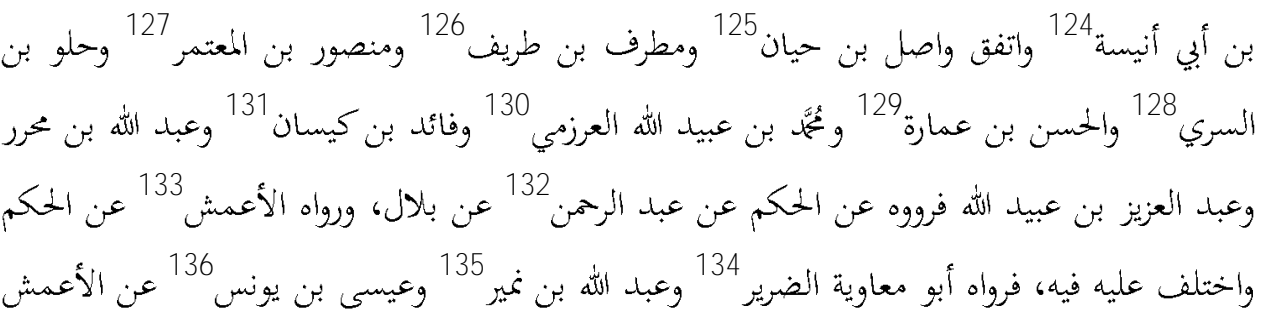 \\
\hline
\end{tabular}

124 _ Al- A sqalani, Ibn H ajar Taqreeb A/- Tahzeeb, P.222.

125 _ Al- A sqalani, Ibn H ajar Taqreeb A/- Tahzeeb, P.579.

هو زيد ابن أبي أنيسة الجزري أبو أسامة،ثقة له أفراد. هو واصل ابن حيان الأحدب الأسدي الكوفن بياع السابري، ثقة ثبت.

126 _ Al-A sqalani, Ibn H ajar, Taqreeb Al- T ahzeeb, P.534.

127 _ Al- A sqalani, Ibn H ajar, Taqreeb A/- T ahzeeb, P.547.

هو مطرف ابن طريف الكويف أبو بكر أو أبو عبد الرمن، ثُّة فاضل. هو منصور ابن المعتمر ابن عبد الله السلمي أبو عتاب الكوين، ثقة ثبت.

128 _ Ibn H ibban, M uhammad, A/- Thiqat,V .6,P.248.

129 _Al-A sqalani, Ibn H ajar, Taqreeb Al- T ahzeeb, P.162.

هو حلو بن السرى من أهل الككوفة، يخطىء ويغرب على قلة روايته.

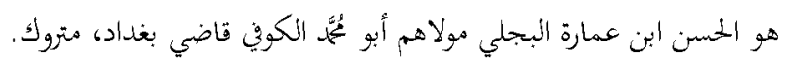

130 _ Al-A sqalani, Ibn H ajar, Taqreeb Al- T ahzeeb, P. 494.

131 _ Al-A sqalani, Ibn H ajar, Taqreeb A/- T ahzeeb, P.444.

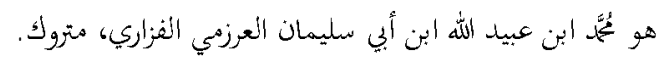

132 A Al-A sqalani, Ibn H ajar Taqreeb Al- Tahzeeb, P.349.

133 _ Al- A sqalani, Ibn H ajar Taqreeb A/- Tahzeeb, P.254.

$$
\text { هو عبد الرمن ابن أبي يلي الأنصاري المدني ثم الكوفي، ثنقة. }
$$

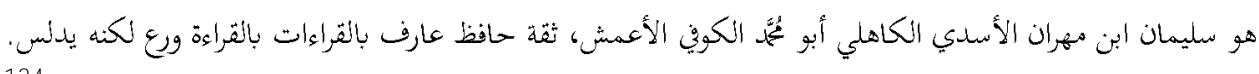

134 _. Al-A sqalani, Ibn H ajar Taqreeb Al- Tahzeeb, P.475.

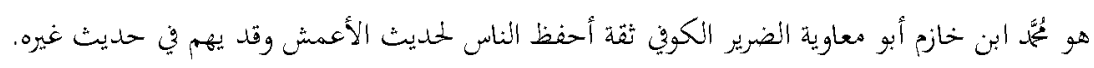

135 _ Al-A sqalani, Ibn H ajar, Taqreeb A/- T ahzeeb, P. 327.

$$
\text { هوعبد الله ابن نمير بنون مصغر الهمداني أبو هشام الكوفي، ثقة. }
$$




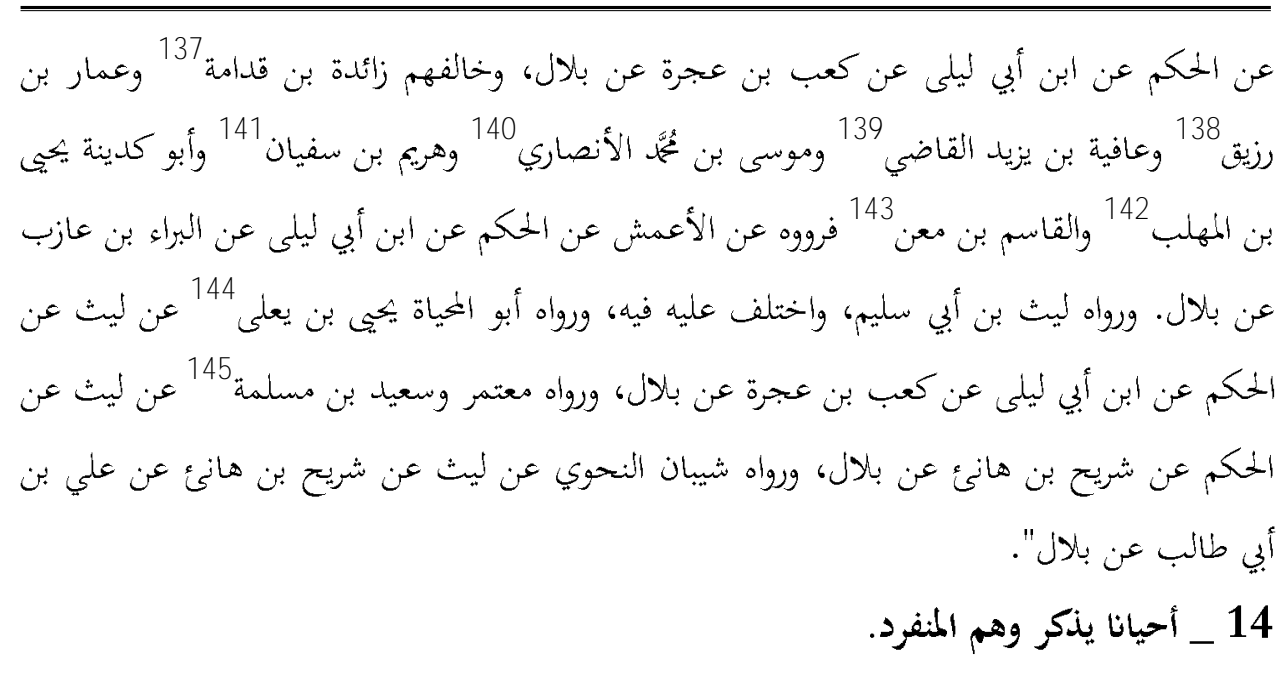

136_Al-A sqalani, Ibn H ajar Taqreeb A/- Tahzeeb, P.441.

137_ Al- A sqalani, Ibn H ajar, Taqreeb Al- T ahzeeb, P.213.

$$
\text { هو عيسى ابن يونس ابن أبي إسحاق السبيعي، ثقة هأمون. }
$$

هو زائدة ابن قداهة الثقفي أبو الصلت الكوفي، ثقة ثبت صاحب سنة.

138 _ Al-A sqalani, Ibn H ajar, Taqreeb Al- T ahzeeb, P.407.

139 _. Al- A sqalani, Ibn H ajar Taqreeb A/- Tahzeeb, P.287.

$$
\text { هو عمار ابن رزيق الضبي أو التميمي أبو الأحوص الكوي،، لا بأس به. }
$$

هو عافية ابن يزيد ابن قيس القاضي الأودي الكوي، صدوق تكلموا فيه.

140 _ Ibn A bi H atim, A bdul R ahman, Al-Jarh wa al- Tadeel,V .8,P160.

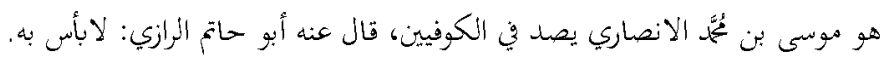

141 _ Al- A sqalani, Ibn H ajar, Taqreeb Al- T ahzeeb, P.571.

142 _ Al-A sqalani, Ibn H ajar Taqreeb A/- Tahzeeb, P.597.

$$
\text { هو هريع ابن سفيان البجلي أبو مُمَّمَ الكوفي، صدوق. }
$$

143 _ Al- A sqalani, Ibn H ajar, Taqreeb Al- T ahzeeb, P.452.

$$
\text { هو يجيى ابن المهلب البجلي أبو كدينة الكوين، صدوق. }
$$

144 _ Al- A sqalani, Ibn H ajar, Taqreeb A/- Tahzeeb, P.598.

145 _ Al- A sqalani, Ibn H ajar Taqreeb Al- Tahzeeb, P.241.

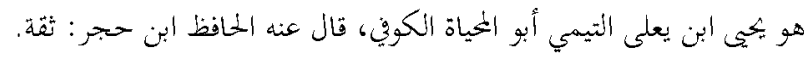

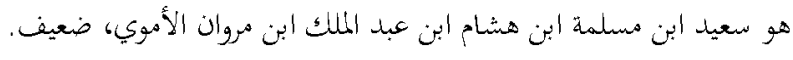




$$
\begin{aligned}
& \text { المثال: أبو إسحاق عن البراء }
\end{aligned}
$$

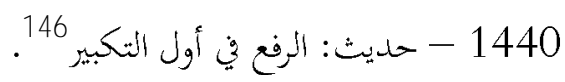

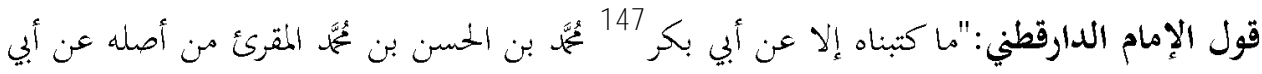

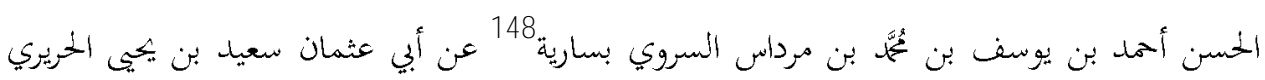

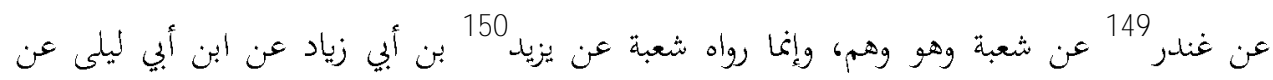

$$
\begin{aligned}
& \text { البراءs 151". } \\
& 15 \text { _ أحيانا يشير إلى طول واختصار الحديث. } \\
& \text { المثال : أبو إسحاق عن البراء } \\
& 1430
\end{aligned}
$$

146 _ Al- M aqasi, M uhammad Ibn A bdul W ahid, A traf $A$ /- Gharaib W al A frad, V.2,P.307, H adith N 0:1440.

147 _K hateeb Al-Baghdadi, A hmed, Tareekh A/-Baghdad, V .2,198.

148 _. H amvi, Y aqoot Ibn A bdullah, Mu'jam al-Buldan, V.3,P.170, Dar Sadir, Beirut, 1995 A.H .

سارية: هي مدينة بطيرستان، والسارية، وهي الأسطوانة،وهي أيضا: السحابة التي تأتي ليلا، وأصله من سرى يسري

149

$$
\text { سرى ومسرى إذا سار ليلا. }
$$

_ Al-A sqalani, Ibn H ajar, Taqreeb A/- T ahzeeb, P.472.

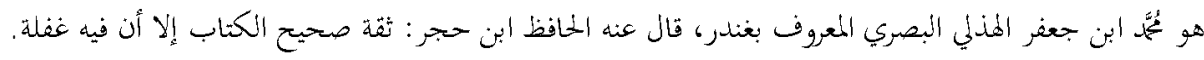

150 _ Al- A sqalani, Ibn H ajar Taqreeb A/- Tahzeeb, P.601.

هو يزيد ابن أبي زياد الهاشي، ضعيف كبر فتغير وصار يتلقن وكان شيعيا.

151 _ Al- M aqasi, M uhammad Ibn Abdul W ahid, A traf Al-Gharaib W al

Afrad, V.2,P.307, H adith N 0:1440.

152_ M uslim,Imam, al- Jami'a al-Sahih,V .4,P.2309, Chapter N 0: 19, D ar

T uraq al- $\mathrm{N}$ ajah, $1422 \mathrm{~A} . \mathrm{H}$, I bn A theer, Al- N ihaya fee G hareeb al-

$\mathrm{H}$ adith, V.2,P.209. 


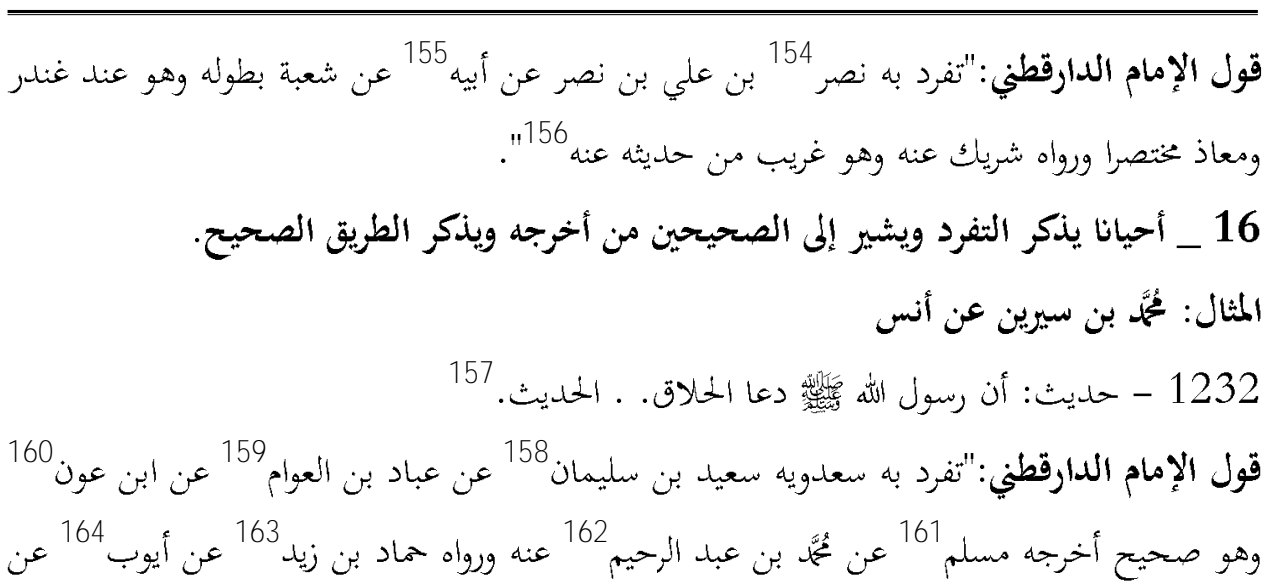

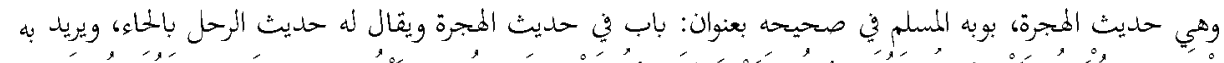

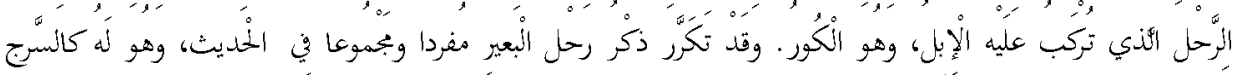

153_ Al- M aqasi, M uhammad Ibn A bdul W ahid, A traf Al- Gharaib W al Afrad, V.2,P.304, H adith N 0:1430.

154 _ Al-A sqalani, Ibn H ajar, Taqreeb A/- T ahzeeb, P.561.

155 هو نصر ابن علي ابن نصر ابن علي الجهضمي، ثقة نبت. - Al-A sqalani, Ibn H ajar, Taqreeb Al- T ahzeeb, P.406. هوعلي ابن نصر ابن علي الجهضمي البصري، تقة. 156 _ Al- M aqasi, M uhammad Ibn Abdul W ahid, A traf Al- Gharaib W al Afrad, V.2,P.304, H adith N 0:1430.

157_Al- M aqasi, M uhammad Ibn A bdul W ahid, A traf A/- Gharaib W al Afrad, V.2,P.231, H adith N 0:1232.

158 _ Al-A sqalani, Ibn H ajar Taqreeb A/- Tahzeeb, P.237.

159 _. Al-A sqalani, Ibn H ajar Taqreeb Al- Tahzeeb, P.290

هو سعيد ابن سليمان الضبي أبو عثمان الواسطي، لقبه سعدويه ثنقة حافظ. هو عباد ابن العوام ابن عمر الكالاي مولاهم أبو سهل الواسطي ثنة.

160 _ Al-A sqalani, Ibn H ajar Taqreeb A/- Tahzeeb, P.317. هو عبد الله ابن عون ابن أرطبان أبو عون البصري ثنقة ثبت فاضل. 161 _. M uslim,Imam, al- Jami'a al-Sahih,V .2,P.947, H adith N 0: 1305. 


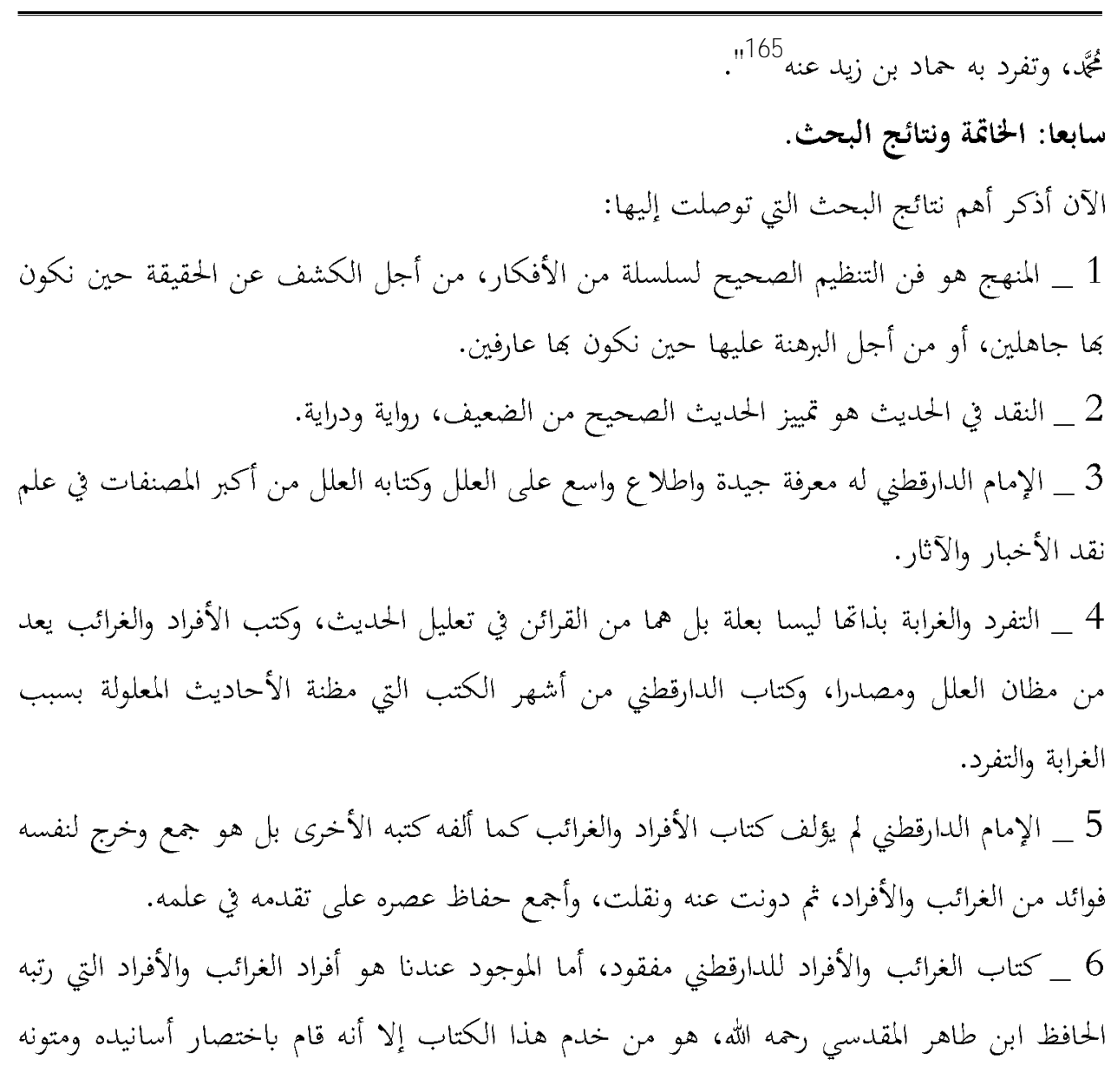

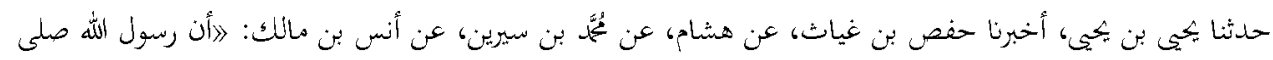

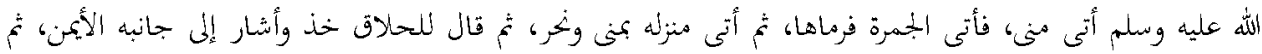
162_ Al-A sqalani, Ibn H ajar, Taqreeb A/- Tahzeeb, P.493.

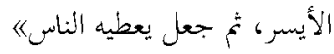

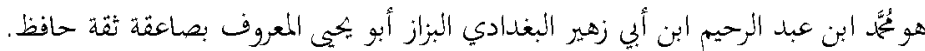
Al-A sqalani, Ibn H ajar Taqreeb A/- Tahzeeb, P.178. هو مماد ابن زيد ابن درهم الأزدي الجهضمي أبو إنماعيل البصري ثقة نبت فقيه. __Al-A sqalani, Ibn H ajar, Taqreeb A/- Tahzeeb, P.117.

هو أيوب ابن أبي تميمة كيسان السختياني أبو بكر البصري ثقة ثبت حجة. 165 _ Al- M aqasi, M uhammad Ibn A bdul W ahid, A traf Al- Gharaib W al Afrad, V.2,P.231, H adith N 0:1232. 
The Scholar Islamic Academic Research Journal

Vol. 7, No. 2 || July-December 2020||P.1-28

https://doi.org/10.29370/siarj/ issue13arabic1

7 حيث لا يذكر سند الحديث كاملا ولايذكر متن الحديث كاملا بل ذكره طرفه منه ثم بعده قول الحافظ 